\title{
12. X-RAY MINERALOGY OF MIOCENE AND OLDER SEDIMENTS FROM DEEP SEA DRILLING PROJECT LEG 78A ${ }^{1}$
}

\author{
Carol J. Pudsey, Department of Geology, University of Leicester ${ }^{2}$
}

\begin{abstract}
One hundred and fifty-three samples of Recent to Late Cretaceous sediments from Sites 541, 542, and 543 were analyzed by X-ray diffraction. The main constituents are quartz, feldspar, clinoptilolite, opal-CT, calcite, and clay minerals (kaolinite, illite, smectite, and palygorskite). Minor components (generally less than $10 \%$ of the total) include amphibole, dolomite, rhodochrosite, chlorite, and mixed-layer clays. Amorphous clays, volcanic glass, and biogenic silica are also present.

Calcite in the form of microfossils is a major component of the Cretaceous and post-Miocene sediment: during the Paleocene to Miocene the sites were below the calcite compensation depth (CCD). Detrital quartz and feldspar increase upsection, recording the passage of the area from ridge crest, through open ocean, to the vicinity of a volcanic arc. Authigenic clinoptilolite may be derived from silicic glass via a smectite intermediate. Opal-CT is present only in Eocene clays, occurring as lepispheres growing on radiolarians. Palygorskite and dolomite, found in the lowest sediments at Site 543 , may be related to hydrothermal activity or alteration of basalt.

The smectite content of the sediments strongly affects their physical properties and their behavior during deformation. Clay mineralogy may therefore be important in the formation of décollement zones.
\end{abstract}

\section{INTRODUCTION}

The three Leg 78A sites are located on the northeast side of the Barbados Ridge in the Central Atlantic, where the Atlantic crest is believed to be underthrusting the Caribbean Plate (Fig. 1). Seismic reflection profiles in the area show layered sediments above ocean crust that dip shallowly westward below a prominent reflector. Above this reflector is a discontinuously reflective unit that thickens westward to constitute the Barbados Ridge. The scientific objectives of Leg 78A included documenting the nature of the discontinuously reflective and layered units and their relationship to each other.

This study is concerned with the sedimentology of the pre-Pliocene muds recovered at Sites 541, 542, and 543 , as revealed by X-ray diffraction (XRD) mineralogy. At the oceanic reference Site 543 the layered sequence is a complete section from Campanian to Holocene. The other two sites contain Miocene to Holocene clays in tectonically repeated slices. The majority of the sediments accumulated by pelagic-hemipelagic settling below the CCD (calcite compensation depth), though some gravity flow deposits rich in foraminifers occur in parts of the Pliocene section (Hemleben, this volume). Although the physical processes of sedimentation are uniform throughout the Leg 78A sites, there is considerable mineralogical variation. Variations in composition can reveal the source of the sediment and affect its physical properties, which in turn determine its behavior during deformation.

\footnotetext{
1 Biju-Duval, B., Moore, J. C., et al., Init. Repts. DSDP, 78A: Washington (U.S. Govt. Printing Office).

2 Address: Department of Geology, University of Leicester, Leicester, United Kingdom.
}

\section{METHODS}

Samples were taken at about 3-m intervals down each core; intervals of poor recovery yielded fewer samples. A few Pliocene and Pleistocene samples were analyzed for the sake of completeness.

\section{Experimental Technique}

A bulk sample was prepared simply by grinding about $0.5 \mathrm{~g}$ of the dried sediment, making it into a sludge with deionized water, and spreading it on a glass slide. A $<2-\mu \mathrm{m}$ fraction was also prepared for more detailed study of the clay minerals. This preparation entailed making a deflocculated suspension with sodium hexametaphosphate and letting the $>2-\mu \mathrm{m}$ fraction settle out; the remaining suspension was centrifuged and the resulting sludge spread evenly over a slide. Each sample was then X-rayed twice-(1) saturated with ethylene glycol and (2) heated to $500^{\circ} \mathrm{C}$. Bulk samples were run from $2 \theta=3^{\circ}$ to $32^{\circ}$ and $<2-\mu \mathrm{m}$ samples from $3^{\circ}$ to $13^{\circ}$. The following instrument settings were used: $\mathrm{Cu} \mathrm{K}_{\alpha}$ radiation, nickel filter, $40 \mathrm{kV} / 20 \mathrm{~m} \AA, 1^{\circ}$ aperture and receiving slits, goniometer speed $2^{\circ} 2 \theta / \mathrm{min}$. Minerals were identified from as many peaks as possible on the diffractograms using the tables given in Brindley and Brown (1980).

\section{Analysis of the Data}

XRD data on peak heights and areas may be used to obtain a semiquantitative estimate of mineralogy by applying a correction factor to the height or area measured for each mineral and assuming that these weighted amounts add up to $100 \%$ of the crystalline fraction. A combination of correction factors given by Rex and Murray (1970), Biscaye (1965), and Mann and Müller (1980a, b, c) is used here (Table 1). I follow Mann and Müller in not correcting peak intensities for the contributions of interfering peaks, because, for a few test samples, the corrections were extremely small. The correction interference of illite (003) with the main quartz peak is not quite so small, but does not significantly alter the trends in abundance reported here. The amount of amorphous material has not been determined quantitatively. Calcite is determined more accurately by carbonate bomb than by XRD (see Wright, this volume), so it was not included in the percentage calculations.

A measure of crystallinity may be obtained by measuring peak width at half height. Poorly crystalline materials, particularly clay minerals, give a broad fuzzy peak rather than a sharp one. Crystallinity of different minerals may be compared using the ratio $H / w$ where $H=$ height and $w=$ width at half height. This approach is not suitable for 


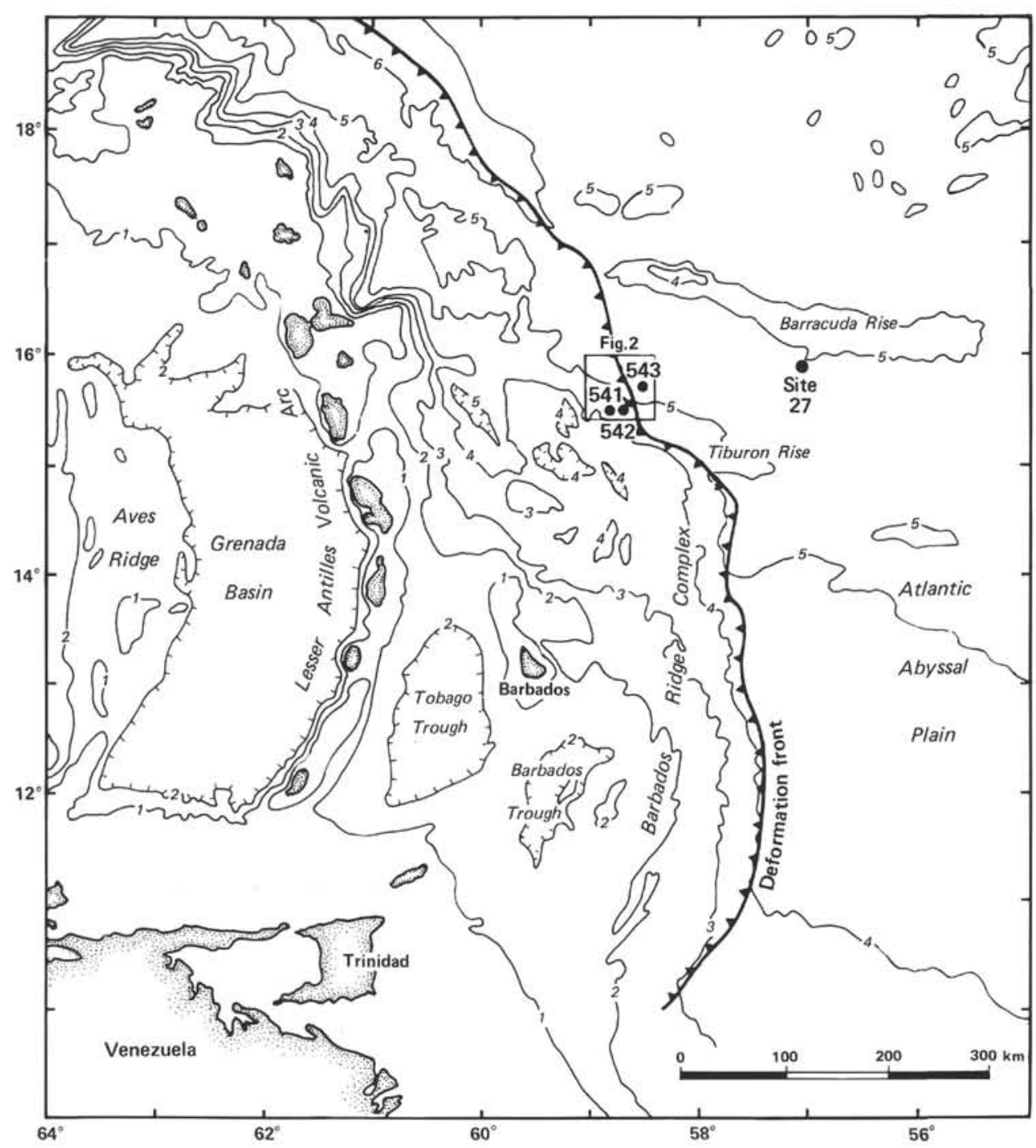

Figure 1. Location of Sites 541, 542, and 543 near the seaward edge of the deformation zone of the Barbados Ridge.

smectite because of high background radiation at low $2 \theta$ angles; the ratio $v / p$ devised by Biscaye (1965) is therefore used (illustrated in Fig. 2D).

Clay minerals are characterized by their strong (001) reflections between $2 \theta=3$ and $14^{\circ}$; in addition (002) peaks occur at higher $2 \theta$ angles. The relative intensity of $(002) /(001)$ may depend on variations in crystallinity and chemistry.

\section{RESULTS}

\section{General Comments}

The sediments recovered during Leg 78A contain the following minerals, in varying proportions: quartz, feldspars, amphibole, clinoptilolite, and opal-CT; the carbonates calcite, rhodochrosite, and dolomite; the clay minerals kaolinite, illite, smectite, chlorite, and palygorskite, with small amounts of mixed-layer clays ( $2 \theta$ from 6.8-7.8 $8^{\circ}$, i.e., $d_{101}$ from $11-13 \AA$ ). These minerals occur together with halite as a contaminant from seawater. In a few samples small amounts of apatite and phillipsite were tentatively identified. Some examples of diffractograms are shown in Fig. 2, and characteristic peaks and correction factors are given in Table 1. In order that my results may be compared with those of all similar studies, the raw data of peak heights and areas are given in Tables 2, 3, and 4. Semiquantitative percentages of minerals, with some measurements of peak sharpness and $(002) /(001)$ peak ratios, are plotted against depth for the three sites in Figures 3 to 8 . The main features of these diagrams are as follows.

\section{Site 541}

Figure 3 illustrates bulk mineralogy for Site 541 . Quartz shows an overall increase upsection, from about $10 \%$ at the base of Tectonic Unit B to 20 to $25 \%$ in the Pliocene and Pleistocene of Tectonic Unit A. Plagioclase fluctuates widely and rather irregularly, shows no overall trend, and does not co-vary with quartz. Feldspar peak sharpness correlates closely with feldspar abundance. Amphibole is a sporadic trace constituent. Total clay (clay minerals in bulk sample) varies mainly from 65 to $80 \%$ but may be as low as $45 \%$ in the Pliocene and $30 \%$ in the feldspathic near-surface sediment. 
Table 1. Characteristic peaks and correction factors for minerals detected in samples from Leg 78A.

\begin{tabular}{|c|c|c|c|c|c|}
\hline \multirow[b]{2}{*}{ Mineral } & \multirow[b]{2}{*}{ Main peak $2 \theta$} & \multirow{2}{*}{\multicolumn{2}{|c|}{ Other peaks }} & \multicolumn{2}{|c|}{$\begin{array}{c}\text { Correction } \\
\text { factors }\end{array}$} \\
\hline & & & & Height & Area $^{a}$ \\
\hline Quartz & $26.7^{\circ}$ & & & 1.3 & \\
\hline Plagioclase & $27.8-28.3^{\circ}$ & 13.8 & $9.4^{\circ}$ & 2.0 & \\
\hline Alkali feldspar & $27.4^{\circ}-27.6^{\circ}$ & & & 2.0 & \\
\hline Amphibole & $10.4^{\circ}$ & & & 2.0 & \\
\hline Clinoptilolite & $9.8^{\circ}$ & $11.2^{\circ}, 2$ & $30.0^{\circ}$ & 2.0 & \\
\hline Opal-CT & $21.8^{\circ}$, broad & & & 1.5 & \\
\hline Calcite & $29.4^{\circ}$ & & & - & \\
\hline Rhodochrosite & $31.4^{\circ}$ & & & 2.0 & \\
\hline Halite & $31.7^{\circ}$ & & & - & \\
\hline \multirow[t]{2}{*}{ Dolomite } & $30.9^{\circ}$ & & & 2.0 & \\
\hline & & & $\begin{array}{l}\text { ain peak } \\
500^{\circ} \mathrm{C}\end{array}$ & & \\
\hline Kaolinite & $12.3^{\circ}$ & $19.9^{\circ}, 24.8^{\circ}$ & Disappears & 4.0. & 2 \\
\hline Illite & $8.9^{\circ}$ & $17.8^{\circ}, 19.9^{\circ}$ & Stable & 8.0 & 4 \\
\hline Smectite glycolated & $5.2^{\circ}$, broad & $19.9^{\circ}$ & Disappears & 10.0 & 1 \\
\hline Chlorite & $6.4^{\circ}$ & $12.3^{\circ}$ & Stable & 4.0 & 2 \\
\hline Palygorskite & $8.4^{\circ}$, broad & $13.7^{\circ}$ & Disappears & 12.0 & 3 \\
\hline $\begin{array}{l}\text { Mixed-layer } \\
\text { clays }\end{array}$ & $\begin{array}{c}6.8-7.6^{\circ} \\
\text { variable }\end{array}$ & & Variable & 4.0 & 2 \\
\hline
\end{tabular}

Note: Feldspar peaks in the range 27.4 to $28.3^{\circ}$ commonly interfere with each other, and positive identification was not always possible; where there were two or more distinct peaks the highest was measured (see Fig. 2A). Smectite is here used as a blanket term for all smectites and illite-smectite interlayers that expand to give a $17 \AA \bar{A}, 5.2^{\circ} 2 \theta$ peak on glycolation. Calcite and halite were not determined quantitatively using xrd; calcite is more accurately determined using carbonate bomb, and halite (probably a contaminant from seawater) was ignored.

a From quartz through dolomite, this factor was not used.

In Tectonic Unit A, calcite is present in only a few samples from the reverse fault at 276 to $200 \mathrm{~m}$ sub-bottom. At $200 \mathrm{~m}$ (lower Pliocene), it increases to about $30 \%$. A similar trend is seen in the lower tectonic unit, calcite being absent altogether below $400 \mathrm{~m}$. Rhodochrosite shows a positive correlation with calcite, being virtually absent below $350 \mathrm{~m}$.

Figure 4 shows the clay mineralogy of $<2-\mu \mathrm{m}$ Site 541 samples. The main feature of this diagram is the large upward decrease in smectite, with a corresponding increase in illite. This trend is clearer in Tectonic Unit B than in A.

Chlorite increases upward in both units, whereas the proportion of kaolinite stays approximately constant at 20 to $25 \%$. The ratio Ka. $(002) /(001)$ shows some upward decrease in each unit, and kaolinite has the sharpest peak of the nonexpandable clays: in general its crystallinity $(H / w)$ decreases upsection, and in the lowest $100 \mathrm{~m}, H / w$ correlates well with abundance. Illite $H / w$ is greatest in the noncalcareous parts of the section. Il. $(002) /(001)$ is always rather low: $0.32 \pm 0.19$ in Unit A and $0.29 \pm 0.14$ in Unit B. Smectite crystallinity $(v / p)$ correlates very clearly with its abundance.

\section{Site 542}

Figures 5 and 6 show Site 542 bulk and clay-fraction mineralogy. Trends similar to those in Tectonic Units A and B at Site 541 are apparent here. Plagioclase content fluctuates less widely, clinoptilolite is absent, and rhodochrosite is less abundant. Calcite decreases sharply below $240 \mathrm{~m}$ sub-bottom but is present in some samples below this depth. Kaolinite is roughly constant at 20 to $30 \%$, and smectite decreases rather abruptly above $260 \mathrm{~m}$.
Crystallinity of kaolinite and smectite decrease upsection. Il. $(002) /(001)$ is $0.26 \pm 0.15$.

\section{Site 543}

Figure 7 shows bulk mineralogy for Site 543. Quartz decreases from 8 to $12 \%$ near the base of the section to a constant 6 to $8 \%$ from 340 to $180 \mathrm{~m}$. Above this depth quartz increases upsection fairly regularly. Plagioclase content fluctuates widely (and also varies in crystallinity) from $200 \mathrm{~m}$ depth to the seafloor. Below $200 \mathrm{~m}$ (Oligocene and older) both alkali feldspar and plagioclase are trace constituents only. Clinoptilolite occurs from 240 to $380 \mathrm{~m}$, a larger depth range than reported from the smear slides (see Site 543 report, this volume) and coinciding with minimum feldspar. The $9.9^{\circ} 2 \theta$ clinoptilolite peak is always sharp and has a rather constant width of 2.5 to $3 \mathrm{~mm}$, so $H / w$ correlates well with abundance. Opal-CT appears first at $340 \mathrm{~m}$ (lower Eocene), where radiolarians also appear, is prominent up to $290 \mathrm{~m}$, and drops to zero at $270 \mathrm{~m}$. Opal-CT and clinoptilolite maxima correspond to total clay minima. Calcite, in widely varying amounts, is present in the lowest $30 \mathrm{~m}$ cored. Dolomite is also a significant component of the basal sediments. Calcite is absent from 380 to $80 \mathrm{~m}$ subbottom and increases upward in the lower Pliocene sediments.

Figure 8 illustrates clay mineralogy of $<2-\mu \mathrm{m}$ Site 543 samples. Kaolinite increases from near zero at the base to more than $50 \%$ at $260 \mathrm{~m}$, then shows a decrease to $12 \%$ at $180 \mathrm{~m}$ and a steady increase to the surface. Illite remains roughly constant at 10 to $20 \%$ except in the lowermost $30 \mathrm{~m}$ and uppermost $90 \mathrm{~m}$. From $200 \mathrm{~m}$ upward smectite decreases, whereas illite and chlorite increase. Palygorskite has a very uneven distribution below $300 \mathrm{~m}$, constituting up to $65 \%$ of the $<2-\mu \mathrm{m}$ fraction.

In general kaolinite has by far the sharpest peak, $H / w$ exceeding 20 in some samples (arbitrary units), and $H / w$ correlates well with abundance in some parts of the section. Illite $H / w$ correlates slightly with abundance above $300 \mathrm{~m}$. Below this depth palygorskite is prominent and the illite and palygorskite peaks are so close together that they merge and their separate widths are not measurable (see Fig. 2). Again smectite $v / p$ correlates with its abundance. Illite $(002) /(001)$ tends to decrease with depth:

\begin{tabular}{rl}
\hline $\begin{array}{c}\text { Depth } \\
(\mathrm{m})\end{array}$ & $\begin{array}{c}\text { Il. } \\
(002) /(001)\end{array}$ \\
\hline $0-100$ & $0.32 \pm 0.11$ \\
$100-200$ & $0.13 \pm 0.19$ \\
$200-300$ & $0.22 \pm 0.2$ \\
$300-410$ & $0.13 \pm 0.12$ \\
\hline
\end{tabular}

\section{Comparison of Age-Equivalent Sections at the Three Sites}

Mineralogy of the Miocene sediments recovered at Sites 541 (two tectonic units), 542, and 543 is compared in Fig. 9. There are no major differences, although mi- 


\section{J. PUDSEY}

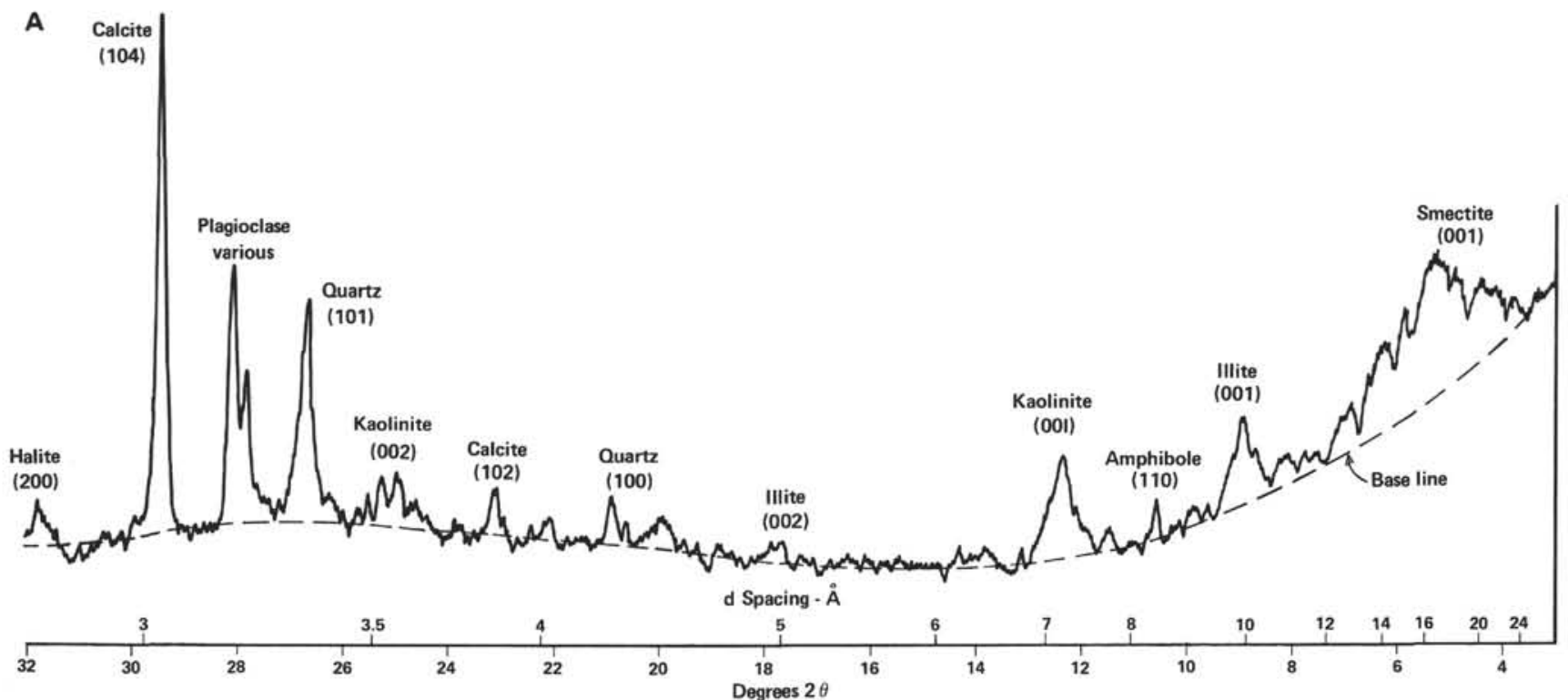

B
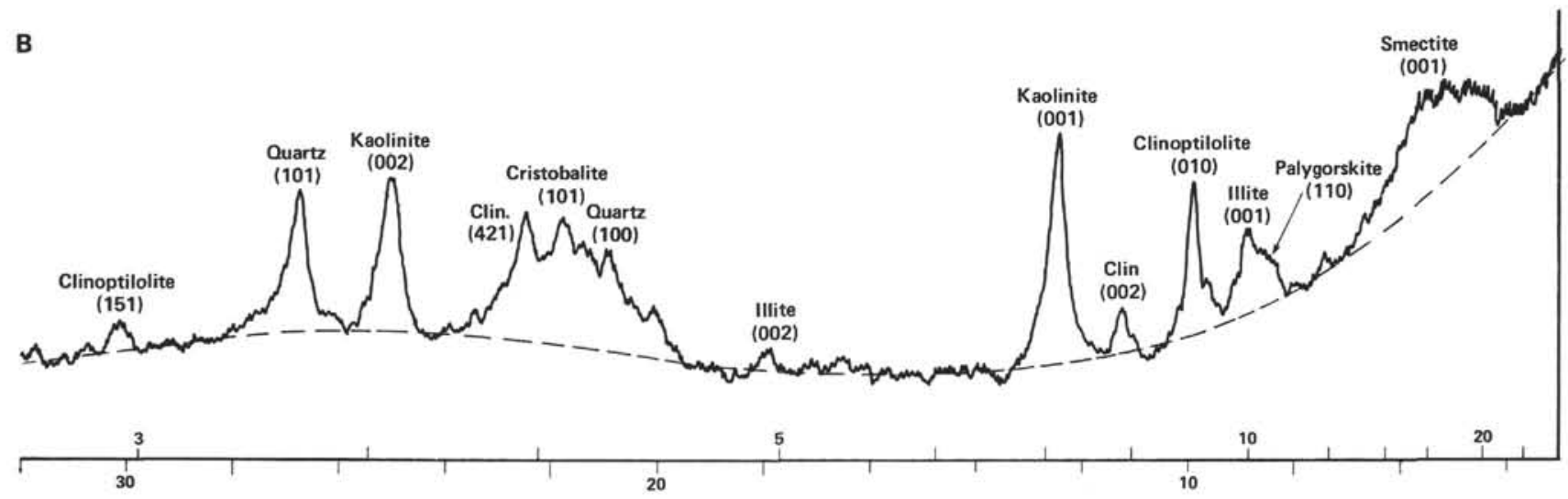

C
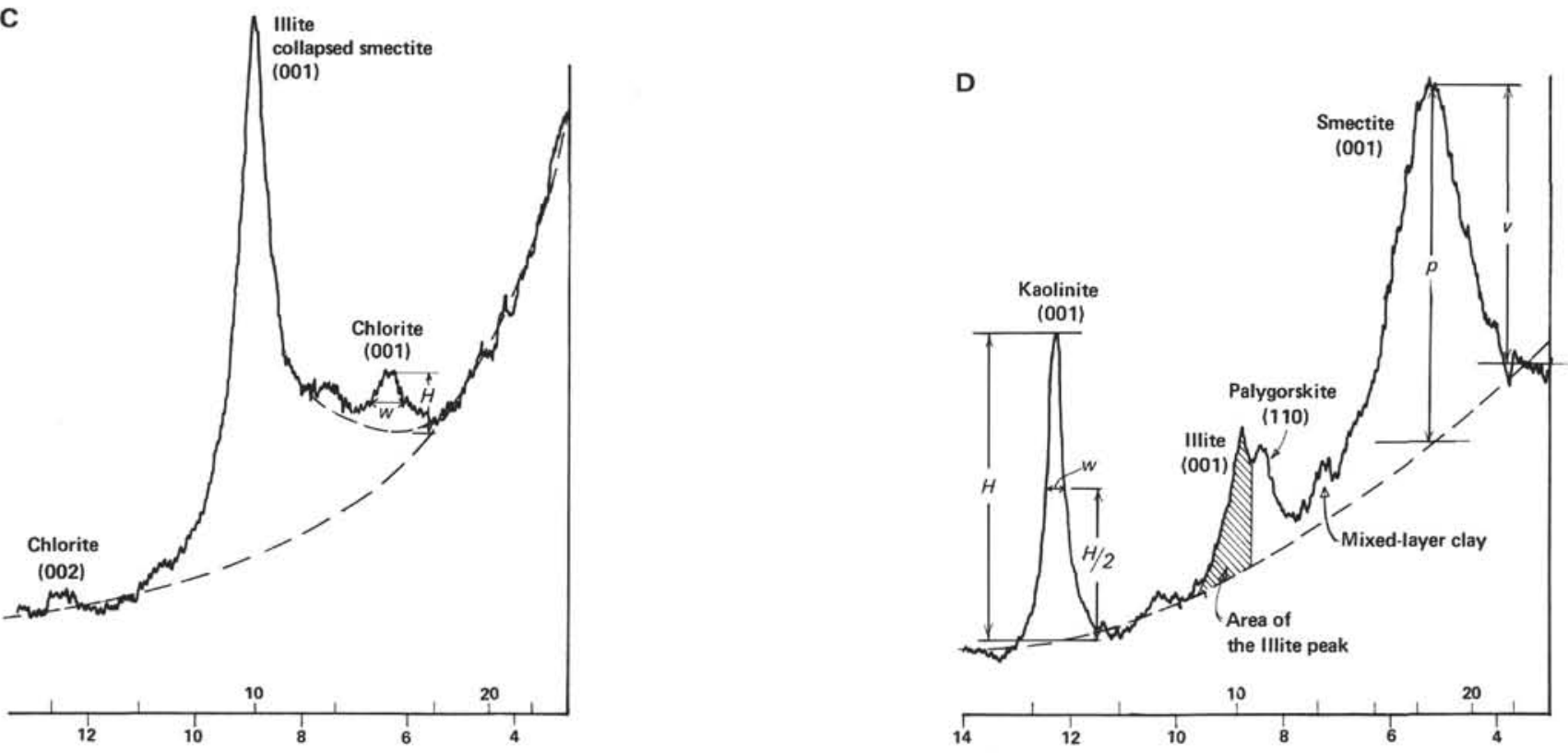

Figure 2. X-ray diffractograms of samples from Leg 78A. (Peak heights and areas are measured in arbitrary units.) A. Sample 542-H2-6, 20 cm bulk sample, glycolated. (Note double plagioclase peak.) B. Sample 543-34-2, $52 \mathrm{~cm}$ bulk sample, glycolated. (Note the very broad opal-CT [cristobalite] peak.) C. Section 543-2,CC $\left(<2-\mu \mathrm{m}\right.$ fraction, heated to $500^{\circ} \mathrm{C}$, showing measurement of $H$ and $w$ for chlorite). D. Section 543A-5,CC $(<2-\mu \mathrm{m}$ fraction, glycolated, showing measurement of $H, w, v$, and $p)$. 
Table 2. Peak heights and areas for minerals of Site 541 samples.

\begin{tabular}{|c|c|c|c|c|c|c|c|c|c|c|c|c|c|c|c|c|c|c|c|c|c|c|c|}
\hline \multirow{2}{*}{$\begin{array}{c}\text { Sample } \\
\text { (core-section, } \\
\mathrm{cm} \text { level) }\end{array}$} & \multicolumn{17}{|c|}{ Bulk samples } & \multicolumn{6}{|c|}{$<2-\mu \mathrm{m}$ samples } \\
\hline & Qz. & Plag. & A.F. & Amp. & Clin. & Phil. & Crist. & $\begin{array}{l}\mathrm{Ka} \text {. } \\
(001)\end{array}$ & $\begin{array}{l}\mathrm{Ka} . \\
(002)\end{array}$ & $\begin{array}{c}\text { Il. } \\
\text { (001) }\end{array}$ & $\begin{array}{l}\text { Il. } \\
(002)\end{array}$ & Sm. & Ch. & Pal. & Ca. & Rh. & Hal. & Ka. & II. & Sm. & Ch. & Pal. & Mixed \\
\hline $2-6,33$ & 25 & 45 & - & 9 & - & - & - & 5 & - & 3 & - & 1.5 & - & - & 4 & - & - & 48 & 55 & 65 & 20 & - & - \\
\hline $4-7,140$ & 30 & 4 & - & - & 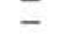 & - & $=$ & 7 & 3 & 7 & $\overline{2}$ & 2 & $\overline{-}$ & $\overline{-}$ & 29 & $=$ & 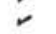 & 51 & 62 & 51 & 22 & - & - \\
\hline $6-1,48$ & 38 & 10 & - & - & - & - & - & 5 & 2 & 5 & - & 3 & 1.5 & 3 & 13 & - & $r$ & 79 & 62 & 147 & 15 & 5 & 23 \\
\hline $8-6,50$ & 22 & 3 & - & - & - & - & - & 8 & 4 & 6.5 & 1.5 & 1 & - & 3 & 72 & 5 & 5 & 49 & 39 & 43 & 13 & 7 & 14 \\
\hline $10-6,50$ & 23 & 15 & - & - & - & - & - & 6 & 2 & 6 & 1 & 2 & 1 & - & 66 & 5 & 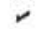 & 39 & 41 & 52 & 16 & - & 5 \\
\hline $12, \mathrm{CC}$ & 18 & 10 & - & - & - & - & - & 5 & - & 2 & - & - & - & - & 25 & - & - & 41 & 22 & 48 & 10 & 5 & 12 \\
\hline $14, \mathrm{CC}$ & 27 & 2 & - & - & - & - & - & 6 & 5 & 6 & 3 & 2.5 & 2 & - & 71 & 4.5 & $=$ & $\begin{array}{l}41 \\
56\end{array}$ & 33 & 124 & 14 & 6 & - \\
\hline $16, \mathrm{CC}$ & 19 & 3 & - & - & - & - & - & 5 & 2 & 2.5 & 2 & - & - & - & 80 & 6.5 & - & 35 & 35 & 110 & 12 & 3 & - \\
\hline $18-6,50$ & 26 & 3 & - & - & - & - & - & 3.5 & 3.5 & 5 & 2 & 1.5 & 1 & - & 82 & 4 & - & 35 & 33 & 61 & 15 & - & - \\
\hline $20-6,50$ & 20 & 5 & - & - & - & - & - & 6 & 3 & 5 & 2 & 2 & 2 & - & 78 & 3.5 & $r$ & 22 & 20 & 59 & 12 & - & 8 \\
\hline $21, \mathrm{CC}$ & 42 & 6 & - & 1 & - & - & - & 14 & 9 & 12 & 4.5 & 6 & 5 & - & 20 & 3 & - & 131 & 123 & 271 & 3 & - & - \\
\hline $22-6,50$ & 29 & 4 & - & 1 & - & - & - & 5.5 & 4.5 & 6 & 2 & 4 & 1.5 & - & 45 & 4 & $=$ & 55 & 64 & 125 & 16 & - & - \\
\hline $23-4,50$ & 18 & 7 & $\therefore$ & 1 & - & - & - & 3 & 2 & 3.5 & 1 & 17 & 2 & - & 15 & - & $r$ & & & & & & \\
\hline $23, \mathrm{CC}$ & 47 & 18 & $(\sim)$ & - & - & - & - & 9.5 & 5 & 7 & 3.5 & 7 & - & - & - & 1 & - & 84 & 73 & 300 & 15 & 8 & - \\
\hline $24, \mathrm{CC}$ & 25 & 5 & - & 4 & - & - & - & 9 & 7 & 7 & 1.5 & 3 & - & 2.5 & 24 & 1.5 & - & 73 & 43 & 223 & 18 & - & - \\
\hline $25-4,50$ & 26 & 8.5 & - & - & - & - & - & 9 & 8 & 14 & 4.5 & 6 & 4 & 3 & 2 & - & - & & & & & & , \\
\hline $25, \mathrm{CC}$ & 30 & 5 & $r$ & 1.5 & - & - & - & 6.5 & 4 & 6 & 1 & 6 & 2 & 2 & 2 & 1.5 & - & 53 & 40 & 222 & 15 & 13 & 7 \\
\hline $26-2,50$ & 24 & 6 & - & - & - & - & - & 10 & 3.5 & 8 & 3 & 10 & 2.5 & - & - & - & - & & & & & & \\
\hline $26-6,20$ & 22 & 8 & - & 1 & - & - & - & 5 & 3 & 3 & 2.5 & 6 & 2 & - & - & - & r & 39 & 49 & 310 & 22 & - & 10 \\
\hline $27-4,50$ & 25 & 4 & - & - & - & - & - & 5 & 2 & 4 & 1 & 9 & 1 & - & - & 2 & - & 24 & 13 & 205 & 6 & - & - \\
\hline $27, \mathrm{CC}$ & 22 & 2 & - & - & - & - & - & 7 & 4.5 & 7 & i & 5 & 2.5 & - & - & 5.5 & - & 66 & 16 & 256 & 5 & - & - \\
\hline $28-2,50$ & 27 & 3 & - & 1 & - & - & - & 12 & 6.5 & 4.5 & i.s & 11 & 5 & - & - & - & - & & & & & & \\
\hline $28, \mathrm{CC}$ & 28 & 1.5 & - & - & - & - & - & 9 & 6 & 4 & 1.5 & 3 & 2 & 2.5 & 18 & 1.5 & - & 66 & 19 & 283 & 8 & 4 & - \\
\hline $29-4,50$ & 25 & 12 & - & 1 & - & - & - & 5.5 & 4 & 4 & i. 5 & 19 & 2 & - & 8 & 1.5 & - & 25 & 16 & 292 & 5 & - & - \\
\hline $29, \mathrm{CC}$ & 28 & 4 & - & - & - & - & - & 10 & 5 & 7 & 2 & 5 & 2 & $=$ & 17 & - & - & 59 & 25 & 276 & 7 & - & 9 \\
\hline $30-4,50$ & 23 & 6.5 & - & - & - & - & - & 7.5 & 2.5 & 4 & 1.5 & 7 & 2 & $\overline{-}$ & 15 & 1 & $\bar{r}$ & 43 & 18 & 248 & 8 & - & - \\
\hline $30-6,50$ & 26 & 21 & - & - & - & - & - & 12 & 5 & 9.5 & 3 & 17 & 3.5 & - & - & - & - & 70 & 10 & 870 & 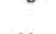 & 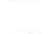 & \\
\hline $30, \mathrm{CC}$ & 26 & 1.5 & - & - & - & - & - & 5 & 4 & 5 & 2 & 3 & 2 & 3 & 68 & 4 & - & 39 & 17 & 71 & 11 & - & - \\
\hline $31-2,50$ & 22 & 9 & $=$ & $\overline{-}$ & $\overline{-}$ & $=$ & $=$ & 7.5 & 3.5 & 5 & 2.5 & 4 & 3 & - & $\begin{array}{l}60 \\
39\end{array}$ & 1.5 & $\bar{z}$ & 8 & $\pi$ & $\pi$ & $n$ & 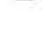 & 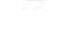 \\
\hline $31-4,50$ & 23 & 2 & $\overline{-}$ & $\overline{-}$ & $\overline{-}$ & $\overline{-}$ & $=$ & 5 & 4 & 6 & 1 & 4 & 1 & $3 . \overline{5}$ & 69 & 4 & $\overline{-}$ & 28 & 10 & 93 & 6 & - & - \\
\hline $31, \mathrm{CC}$ & 20 & 2 & $=$ & $=$ & $=$ & $=$ & $=$ & 9 & 3.5 & 7 & 2.5 & 4 & 2.8 & - & 60 & 4 & $\overline{-}$ & 37 & 30 & 102 & 6 & 6 & - \\
\hline $32, \mathrm{CC}$ & 17 & 2 & $\overline{-}$ & $\bar{z}$ & $\bar{z}$ & $\bar{z}$ & $\bar{z}$ & 5 & 3 & 4 & 1 & $\begin{array}{l}4 \\
5\end{array}$ & 2.8 & $\bar{z}$ & 85 & 5 & $\bar{z}$ & 30 & 38 & 58 & 11 & 7 & $\overline{-}$ \\
\hline $33, \mathrm{CC}$ & 26 & 1 & - & - & - & - & - & 6.5 & 3 & 7 & i.s & 7 & 2 & - & 63 & 3 & - & 38 & 52 & 80 & 23 & - & - \\
\hline $34, \mathrm{CC}$ & 30 & 2 & $=$ & 1 & $\bar{z}$ & $=$ & $\bar{z}$ & $\begin{array}{l}\text { l.s } \\
6\end{array}$ & 4.5 & 8 & 3 & 5 & 2 & $\Xi$ & 59 & 3 & $\bar{z}$ & 45 & 60 & 69 & 18 & $\bar{z}$ & 8 \\
\hline $35-4,44$ & 24 & 17 & - & - & - & - & - & 6.5 & 3 & 7 & 2 & 10 & 3 & 2 & 43 & 2 & $\bar{c}$ & 30 & 16 & 345 & $\begin{array}{l}16 \\
16\end{array}$ & - & - \\
\hline $35, \mathrm{CC}$ & 31 & 5 & - & - & - & - & - & 8.5 & 3 & 6 & 3 & 4 & 2.5 & 1 & 46 & 3 & - & 71 & 33 & 166 & 16 & 4 & 6 \\
\hline $36-2,50$ & 33 & 9.5 & - & - & - & - & - & 20 & 10.5 & 17 & 4.5 & 5 & 4 & i & 18 & 1 & - & & & & & & \\
\hline $36, \mathrm{CC}$ & 32 & 20 & - & - & - & - & - & 11 & 6.5 & 9 & 3 & 7 & 3 & $i$ & 21 & - & - & 64 & 30 & 240 & 17 & 4 & - \\
\hline $37-2,50$ & 27 & 2.5 & - & - & - & - & - & 15 & 6.5 & 12.5 & 5.5 & 4 & 4 & - & 30 & - & - & 63 & 33 & 121 & 23 & 5 & - \\
\hline $37-4,50$ & 34 & 3.5 & - & - & - & - & - & 15 & 10 & 12.5 & 4.5 & 3.5 & 4 & 2 & 11.5 & 1.5 & - & 147 & 121 & 419 & - & 14 & 9 \\
\hline $37-6,49$ & 36 & 4 & $r$ & - & - & - & - & 15.5 & 9.5 & 14 & 4.5 & 12 & 3 & - & - & - & - & & & 719 & & & \\
\hline $38-4,50$ & 26 & 3 & - & 1 & - & - & - & 7 & 5 & 7 & 2 & 3 & 2 & - & - & - & - & & & & & & \\
\hline $38, \mathrm{CC}$ & 26 & 5 & - & 1 & - & - & - & 9 & 6 & 8 & 2.5 & 6 & 4 & - & - & - & 1 & 51 & 23 & 246 & 16 & - & - \\
\hline $39-2,50$ & 23 & 16 & - & - & - & - & - & 9 & 6.5 & 9 & 2 & 2.5 & 3 & - & 11 & 1 & - & & & & & & \\
\hline $39-4,50$ & 32 & 26 & - & - & - & - & - & 6 & 6.5 & 6 & 2 & 7 & 4 & - & 8 & 1 & r & 30 & 16 & 237 & 13 & - & - \\
\hline $40-2,52$ & 26 & 4 & $r$ & - & - & - & - & 8 & 7 & 7 & 2.5 & 5.5 & 2.5 & 3 & - & - & - & 54 & 30 & & 6 & - & 4 \\
\hline $40, \mathrm{CC}$ & 27 & 4 & $r$ & - & - & - & - & 11 & 6 & 7 & 3 & 5 & 4 & - & - & - & - & 34 & 21 & 276 & 7 & - & - \\
\hline $41, \mathrm{CC}$ & 23 & 10 & $r$ & - & - & - & - & 9 & 8 & 2 & - & 8 & 2 & - & - & - & - & 63 & 21 & 229 & 6 & - & - \\
\hline $42-7,70$ & 28 & 11 & - & - & - & - & - & 10 & 7.5 & 4 & - & 7 & 2 & 4 & - & - & - & 38 & 7 & 260 & 10 & - & - \\
\hline $43-3,50$ & 22.5 & 7 & - & - & - & - & - & 7 & 6.5 & 6 & - & 6 & 2.5 & - & - & - & - & & & & & & \\
\hline $43-6,50$ & 27 & 9 & $r$ & 2 & - & - & - & 9 & 5.5 & 5 & 1 & 14 & 2.5 & - & - & 1 & - & 32 & 10 & 377 & 10 & 3 & - \\
\hline $44, \mathrm{CC}$ & 19 & 3 & $r$ & $i$ & - & - & - & 6 & 2.5 & 3.5 & i.s & 7 & - & - & - & - & - & 23 & 7 & 373 & 6 & - & - \\
\hline $46, \mathrm{CC}$ & 21 & 5 & 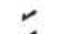 & - & - & - & - & 6 & 4.5 & 3 & $i$ & 7 & 2 & - & - & - & - & 18 & 6 & 323 & 7 & - & - \\
\hline $47-1,49$ & 21 & 14 & $r$ & - & - & - & - & 5 & 4. & 5 & i.5 & 11 & 2.5 & - & - & - & $r$ & & & & & & \\
\hline $47-2,95$ & 22 & 33 & $(r)$ & - & 2 & - & - & 6 & 2.5 & 5 & 2 & 9.5 & 2.5 & - & - & - & - & & & & & & \\
\hline $47, \mathrm{CC}$ & 26 & 22 & 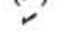 & - & 2.5 & - & - & 11 & 9 & 9 & 2 & 17 & - & - & - & - & - & 38 & 8 & 337 & 7 & - & - \\
\hline $48-1,48$ & 24 & 18 & $r$ & - & - & - & - & 3.5 & 2.5 & 3 & 2 & 6.5 & 2 & 2.5 & - & - & $=$ & & . & & & & \\
\hline $48-3,52$ & 22 & 41 & $r$ & - & - & - & - & 7.5 & 4.5 & 4.5 & 1.5 & 9 & 1.5 & - & - & - & - & & & & & & \\
\hline $48-4,50$ & 29 & 28 & (r) & 1.5 & - & - & - & 9.5 & 6 & 10.5 & 1.5 & 9 & 3 & 3 & - & - & 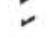 & & & & & & \\
\hline & 3 & 25 & - & 1 & - & - & - & - & - & 2.5 & - & 7 & 2 & - & - & 1.5 & - & & & & & & \\
\hline & 16 & 19 & - & - & - & - & - & 2.5 & 1.5 & 5 & 2 & 10 & 1.5 & - & - & - & - & & & & & & \\
\hline & 27 & 14 & $r$ & 1 & - & - & - & 12 & 7. & 6 & 1 & ic & - & - & - & - & - & 42 & 12 & 398 & & & 5 \\
\hline & 26 & 12 & $r$ & - & - & - & - & 8 & 4.5 & 6 & 1 & 15 & 3 & - & - & 4 & - & 29 & 7 & & - & - & - \\
\hline & 23 & 12.5 & $(r$ & - & 4 & - & - & 13 & 9 & 11 & 2.5 & 10 & 1.5 & 1.5 & - & - & - & 98 & 27 & 528 & 5 & 6 & - \\
\hline 50-4, & 27 & 5.5 & ( & - & 4.5 & - & - & 21 & 10 & 11 & 2.5 & 8.5 & 1 & 2 & - & - & - & & & & & & \\
\hline $50, \mathrm{CC}$ & 25 & 7 & (r) & - & 4.5 & - & - & 27 & 17 & 8 & 2 & 14 & 3 & 2 & - & - & - & 106 & 11 & 382 & - & - & - \\
\hline
\end{tabular}

nor fluctuations are apparent in feldspar and total clay in the bulk samples.

\section{DISCUSSION}

\section{General Remarks}

The sediments cored during Leg 78A contain both detrital and authigenic components. Detrital minerals are biogenic (calcite) and terrigenous (quartz, feldspar, amphibole, kaolinite, illite, chlorite, mixed-layer clay minerals, and some smectite). Authigenic minerals include zeolites, cristobalite, dolomite, some smectite, and palygorskite. Overall, the section cored at Site 543 records the passage of this site from ridge crest, through open ocean, to its present position close to an island arc. The shorter sections recovered at Sites 541 and 542 provide some more detail on the upper part of the succession. There is little variation in the Miocene and lowest Pliocene between sites (Fig. 9). This is to be expected, because the sites are relatively close to each other and were far from island arc or continent sources during the Miocene and early Pliocene (see Fig. 1).

The mineralogy of deep ocean sediments approaching a subduction zone has potentially important effects on their physical properties and hence their behavior during deformation. 
Table 3. Peak height and areas for minerals of Site 542 samples.

\begin{tabular}{|c|c|c|c|c|c|c|c|c|c|c|c|c|c|c|c|c|c|c|c|c|c|c|c|}
\hline \multirow{2}{*}{$\begin{array}{l}\text { Sample } \\
\text { (core-section, } \\
\text { cm level) }\end{array}$} & \multicolumn{17}{|c|}{ Bulk samples } & \multicolumn{6}{|c|}{$<2-\mu \mathrm{m}$ samples } \\
\hline & Qz. & Plag. & A.F. & Amp. & Clin. & Phil. & Crist. & $\begin{array}{c}\mathrm{Ka} . \\
(001)\end{array}$ & $\begin{array}{l}\mathrm{Ka} . \\
(002)\end{array}$ & $\begin{array}{l}\text { Il. } \\
(001)\end{array}$ & $\begin{array}{l}\text { Il. } \\
(002)\end{array}$ & Sm. & Ch. & Pal. & Ca. & Rh. & Hal. & Ka. & II. & Sm. & Ch. & Pal. & Mixed \\
\hline $\mathrm{A} 1, \mathrm{CC}$ & 23 & 3.5 & $r$ & 1.5 & - & - & - & 10 & 7 & 13.5 & 4.5 & 7.5 & - & $r$ & 44 & - & $r$ & 48 & 27 & 115 & 6 & - & - \\
\hline $\mathrm{AH} 2-6,20$ & 16 & 19.5 & - & 3 & - & - & - & 7.5 & 4.5 & 6.5 & 1.5 & 10 & 1.5 & - & 38 & - & $r$ & 31 & 14 & 232 & 13 & - & - \\
\hline $1-2,50$ & & & & & & & & & & & & & & & & & & 20 & 7 & 97 & - & - & 2 \\
\hline $1, \mathrm{CC}$ & 22 & 2 & - & - & - & - & - & 8.5 & 4 & 9 & 3 & 5 & 3 & - & 71 & 3 & r & 19 & 12 & 82 & 6 & - & - \\
\hline $2, \mathrm{CC}$ & 27 & 7.5 & - & 1 & - & - & - & 9 & 5.5 & 6.5 & 1.5 & 4 & 2.5 & 2.5 & 48 & 2.5 & $r$ & 26 & 6 & 89 & 11 & - & - \\
\hline $3, \mathrm{CC}$ & 27 & 1.5 & $r$ & - & - & - & - & 10 & 6 & 9 & 1.5 & 5 & 3 & - & 42 & 2 & - & 32 & 25 & 125 & 4 & - & - \\
\hline $4, \mathrm{CC}$ & 37 & 6 & $(r)$ & - & - & - & - & 6.5 & 5 & 7 & 2.5 & 4.5 & - & $(r)$ & - & - & $r$ & 58 & 39 & 251 & 16 & - & - \\
\hline $\mathrm{A} 2, \mathrm{CC}$ & 28 & 3.5 & $r$ & - & - & - & - & 8 & 6.5 & 11 & 3.5 & 5.5 & 3.5 & - & - & 1.5 & - & 72 & 35 & 267 & 14 & - & 5 \\
\hline A3-4, 50 & - & - & - & - & - & - & - & - & - & - & - & - & - & - & - & - & - & 51 & 21 & 332 & 7 & - & - \\
\hline $\mathrm{A} 3, \mathrm{CC}$ & 18 & 6.5 & - & 1 & - & - & - & 4 & 3.5 & 3 & 2 & 5.5 & - & - & 5 & - & - & 16 & 6 & 244 & 15 & - & - \\
\hline $\mathrm{A} 4, \mathrm{CC}$ & 19.5 & 5 & - & - & - & - & - & 3 & 2.5 & 2 & - & 6 & - & - & - & - & $r$ & 21 & 3 & 202 & 2 & - & - \\
\hline A5,CC & 22 & 3 & $r$ & 2 & - & - & - & 6 & 2.5 & 3.5 & 1 & 4 & 2 & 2 & - & - & - & 17 & 2 & 174 & 5 & - & - \\
\hline A6,CC & 22 & 3 & - & - & - & - & - & 8 & 4.5 & 5 & - & 6.5 & 3 & 2.5 & 2 & 1.5 & $r$ & 26 & 3 & 157 & - & - & - \\
\hline A7-4, 50 & 25 & 5 & $r$ & 1.5 & - & - & - & 14 & 9 & 4.5 & 1 & 8.5 & 2.5 & 4 & - & 1.5 & $r$ & 54 & 10 & 257 & 10 & - & - \\
\hline A8-2, 50 & 23 & 8 & 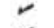 & - & - & - & - & 16 & 11.5 & 8.5 & 1.5 & 12 & 2.5 & 8 & - & - & - & 49 & 9 & 311 & 12 & - & - \\
\hline A9,CC & 26 & 3.5 & $r$ & 1.5 & - & - & - & 16.5 & 10 & 10 & 2 & 11.5 & 3.5 & 5.5 & 13 & - & - & 57 & 11 & 311 & 6 & 3 & - \\
\hline Al $0-4,64$ & 22 & 6 & $\sim$ & 1 & - & - & - & 10 & 4.5 & 4.5 & 1.5 & 9.5 & 3.5 & 4 & - & - & - & 23 & 5 & 251 & 8 & - & - \\
\hline
\end{tabular}

Note: See Table 2 note for explanation of symbols and abbreviations.

\section{Detrital and Authigenic Minerals}

\section{Calcite}

The majority of Paleogene and Miocene sediments are carbonate-free, having been deposited below the CCD. However, calcite in the form of nannofossils and foraminifers is a major component of the Pliocene and younger sediments. This distribution is a simple consequence of an abrupt drop in the CCD in the area at the end of the Miocene (Hemleben, this volume); it does not imply any significant vertical movement of the seafloor at that time. Calcareous sediments do occur in the predominantly clay sections of the Miocene at Sites 541 and 542 . This may be a consequence either of gravity sliding or tectonic repetition of units that are too thin and of too short a time span to detect using biostratigraphy. There is at least one minor repetition at the base of Tectonic Unit A at Site 541: a nannofossil zone is repeated (Bergen, this volume), some calcite appears in the sediment, and the upward decrease in smectite is interrupted (Figs. 4, 9).

Calcite forms up to $50 \%$ of the basal sediments deposited near the ridge crest, again mainly in the form of nannofossils showing evidence of dissolution (Hemleben, this volume). The transition from calcareous to noncalcareous sediments reflects the cooling of the crust and its subsidence below the CCD. Backtracking the site using the method of Berger and Winterer (1974) yields a paleo-depth of about $4000 \mathrm{~m}$ for the Eocene CCD in the Central Atlantic.

\section{Quartz}

Terrigenous quartz may be derived from three sources: the Lesser Antilles arc, the South American continent, and the African continent. The increase in quartz upsection at Site 543 implies that arc sources were the most important, with quartz content increasing as the site approached the arc. During the deposition of the basal sediments (Campanian), the Atlantic Ocean was only two-thirds of its present width, and it is possible that wind-blown continental dust from North Africa contributed to the sediments. However, the Sahara Desert, an important present-day source of airborne dust, did not develop before the late Miocene (van Zinderen Bakker, 1978). Another source of quartz may be recrystallization of opal-CT (see later discussion).

\section{Feldspar and Amphibole}

The decrease in plagioclase and (less clearly) amphibole downsection at Site 543 may be attributed to the greater distance of this site from the volcanic arc in the Paleogene and perhaps to a less explosive style of volcanism (or 2 more tholeiitic sequence of differentiation) at those times. Thin ash beds are common in the Neogene (see Natland, this volume) and are mixed with the surrounding sediment to a varying degree by bioturbation and drilling disturbance. Wide variations in feldspar and amphibole content at all sites may result partly from our having taken small samples. If the samples had been composited from a whole core or section, the local effects of ash beds might have been smoothed out. Small amounts of alkali feldspar in the basal sediments may be related to ridge-crest volcanism or to alteration of calcic plagioclase (see later discussion). In contrast to quartz, feldspar is not a common component of windborne pelagic sediment, being mechanically and chemically less stable.

\section{Detrital Clay Minerals}

Kaolinite, illite, chlorite, and the few mixed-layer clays are probably derived both from volcanic-arc and continent sources. The Tiburon Rise has partly shielded the Leg 78A area from South American continental sediment (see also Wright, this volume), but these clay minerals are also present in weathered tropical soils on volcanic islands and in sediment aprons derived therefrom (e.g., Lonsdale, 1975). The generally low proportion of chlorite is consistent with the low-latitude setting (Biscaye, 1965). The abundant and well-crystallized kaolinite found in the Oligocene and Eocene at Site 543 may be partly authigenic or neoformational in origin. Further discussion of this point occurs in the chapter by Latouche and Maillet (this volume). Low (002) peak intensity of illite compared with (001) intensity implies that 
Table 4. Peak height and areas for minerals of Site 543 samples.

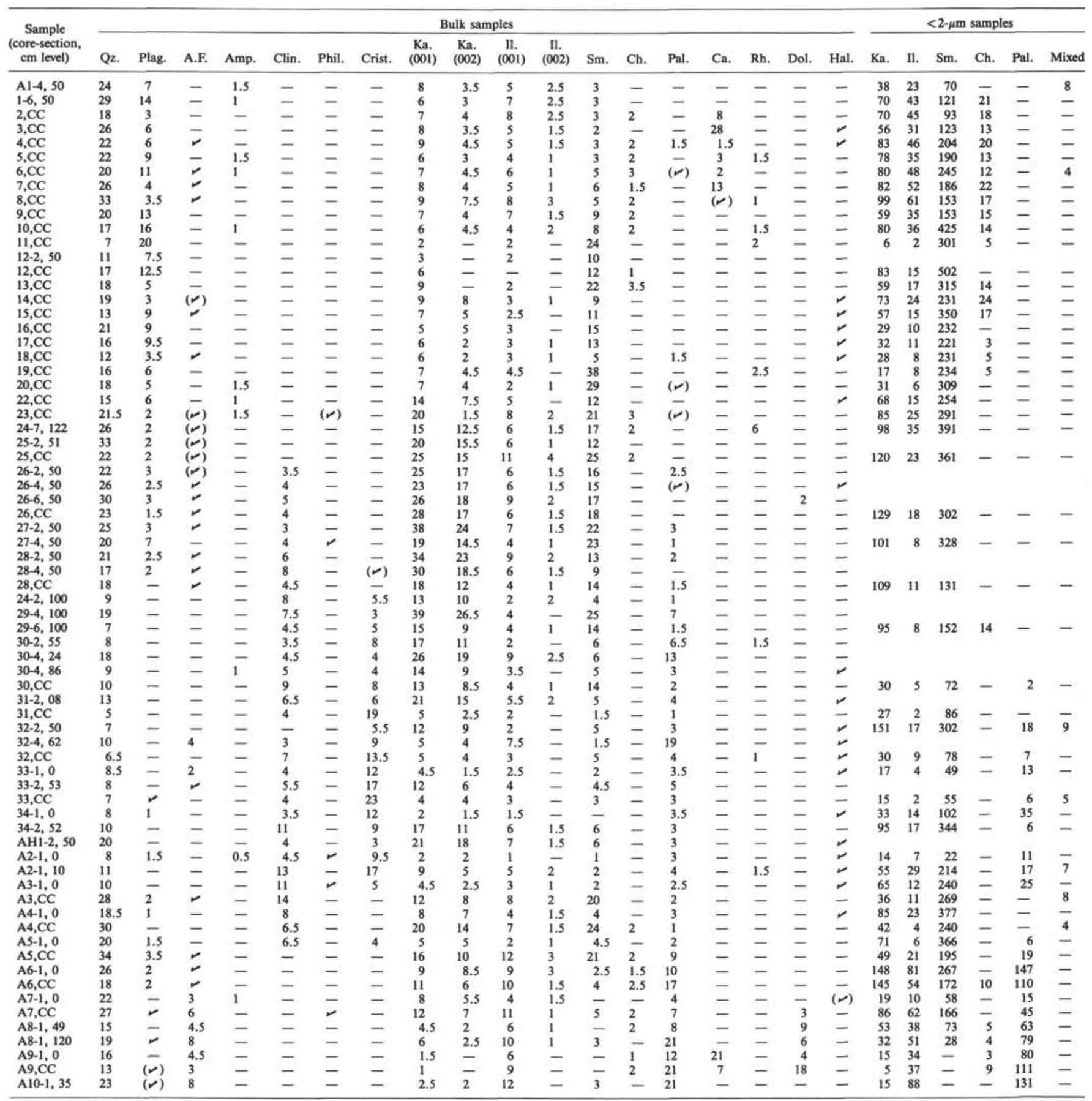

Note: See Table 2 note for explanation of symbols and abbreviations.

the $\mathrm{Al}: \mathrm{Fe}$ ratio must be high, between 3:1 and 4:0 (Brindley and Brown, 1980, p. 338). It is difficult to estimate the amount of disordering of kaolinite in the presence of other clay minerals, because the relevant peaks at about $19.8^{\circ} 2 \theta$ overlap. In a few samples the $19.8^{\circ}$ peak is strong and asymmetrical, and the kaolinite group mineral may in fact be nearer structurally to halloysite than kaolinite sensu stricto.

\section{Zeolites}

Clinoptilolite is the only zeolite positively identified. One might have expected phillipsite as an alteration prod- uct of vitric basaltic ashes, but it is difficult to detect in the presence of plagioclase and kaolinite. It may be present in some samples (see Figs. 3 and 7). Clinoptilolite, the high-silica member of the heulandite group, is a characteristic alteration product of rhyolitic glasses in an alkaline environment (Hay, 1966; von Rad and Rösch, 1972). The initial alteration of glass to smectite may be an important factor in providing the chemical environment (high pH and high $\mathrm{SiO}_{2}$ ) suitable for subsequent zeolite formation (Hay, 1966, p. 81). In DSDP cores clinoptilolite is more common in calcareous sediments, though it is not unknown in clays (Stonecipher, 


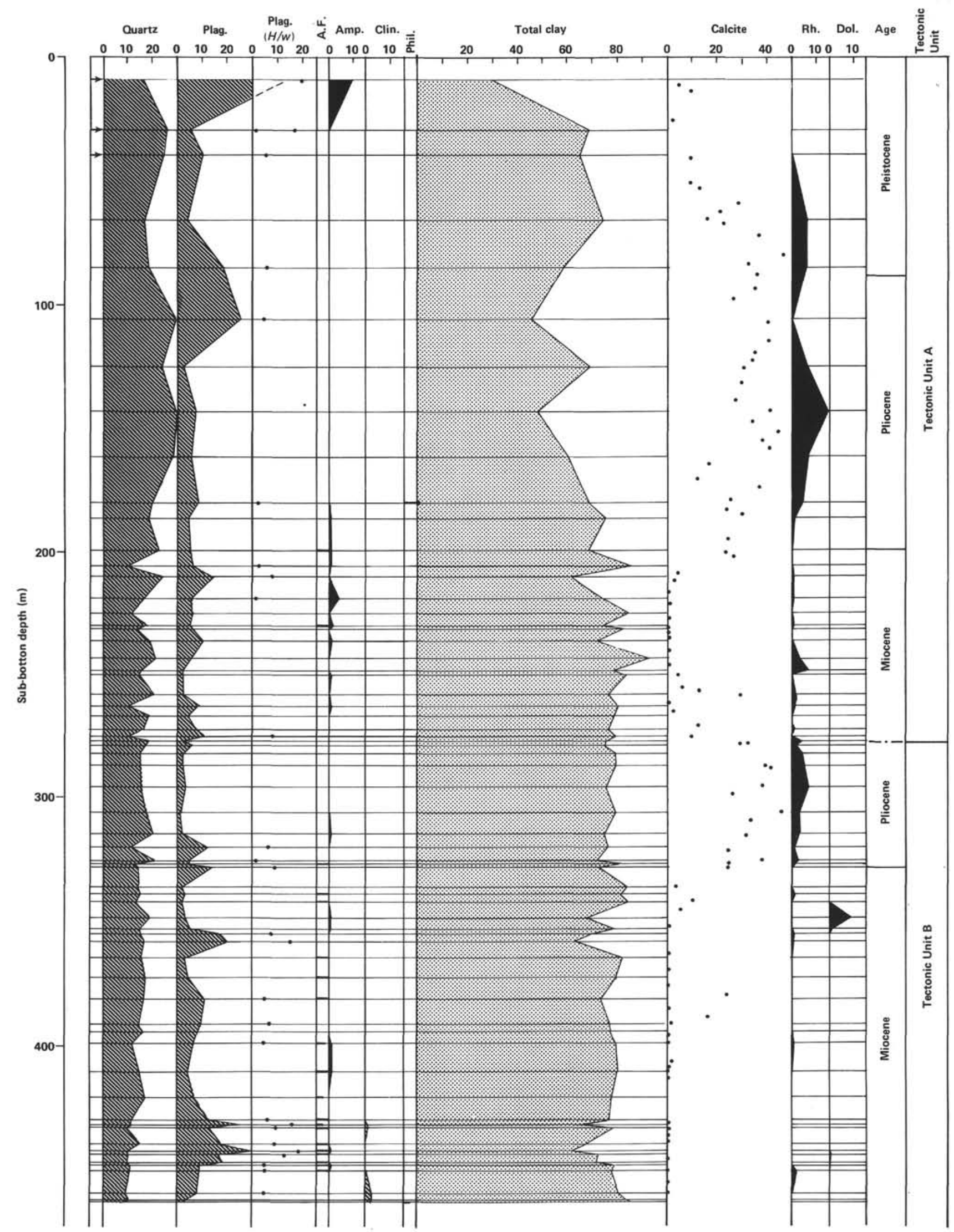

Figure 3. Site 541, mineralogy of bulk samples (\%). (Horizontal lines represent sample positions. Calcite percentages are from carbonate bomb. Percentages of remaining minerals assume no calcite [i.e., totalled to $100 \%$ ]. Plag. $=$ plagioclase, A.F. $=$ alkali feldspar, Amp. $=$ amphibole, Clin. = clinoptilolite, Phil. = phillipsite, Rh. = rhodochrosite, Dol. = dolomite. For alkali feldspar and phillipsite, a bar across the column indicates presence [these minerals could not be determined quantitatively]. A half bar indicates minor presence [tiny peaks].) 


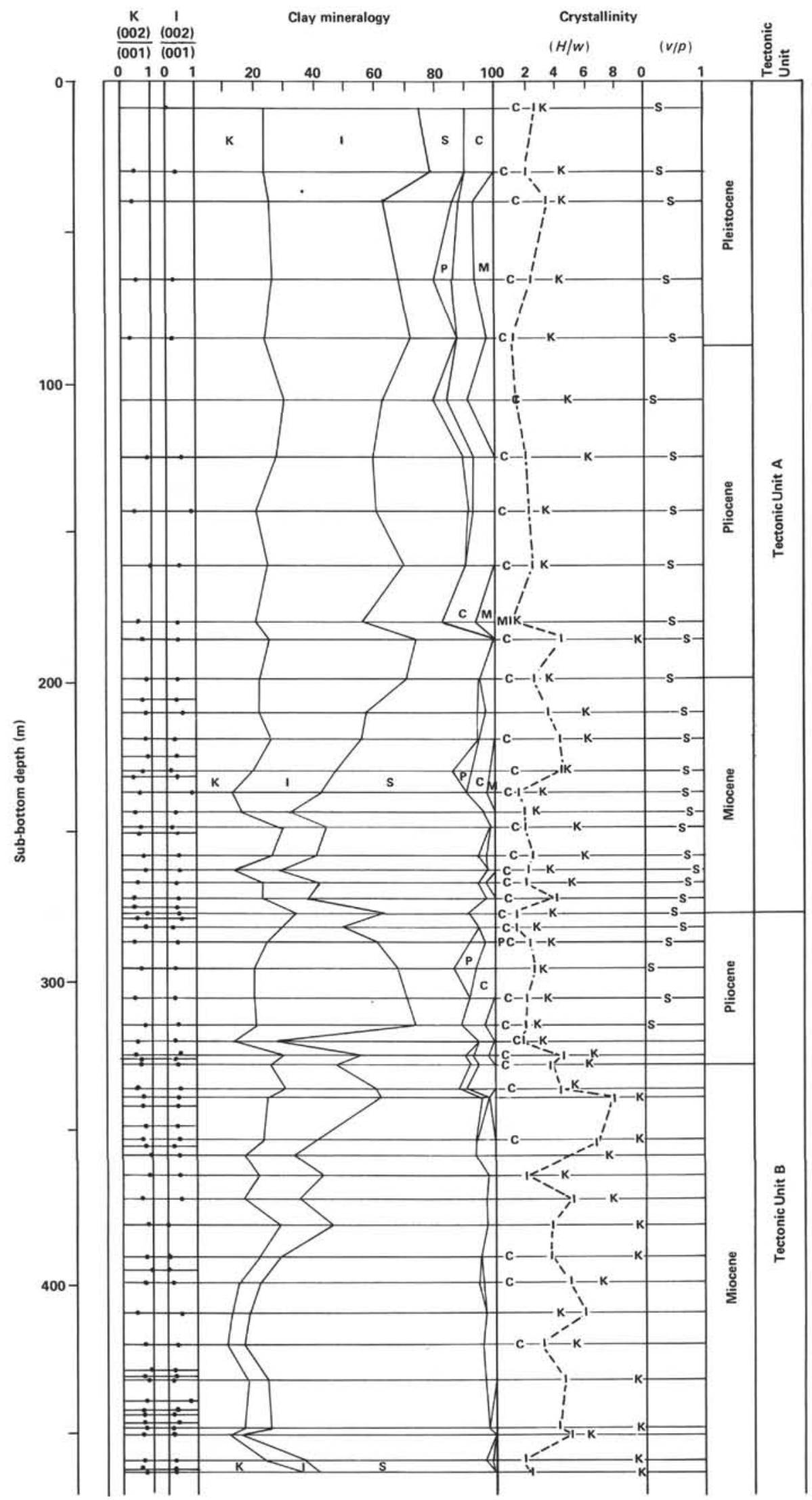

Figure 4. Site 541, mineralogy of $<2-\mu \mathrm{m}$ samples. (Horizontal lines represent sample positions. $(002) /(001)$ peak ratios calculated from bulk mineralogy diffractograms. $\mathrm{K}=$ kaolinite, $\mathrm{I}=$ illite, $\mathrm{S}=$ smectite, $\mathrm{C}=$ chlorite, $\mathrm{P}=$ palygorskite, $\mathrm{M}=$ mixed-layer clays. $H, w, v$, and $p$ are defined in the text and shown in Figure 2D.) 


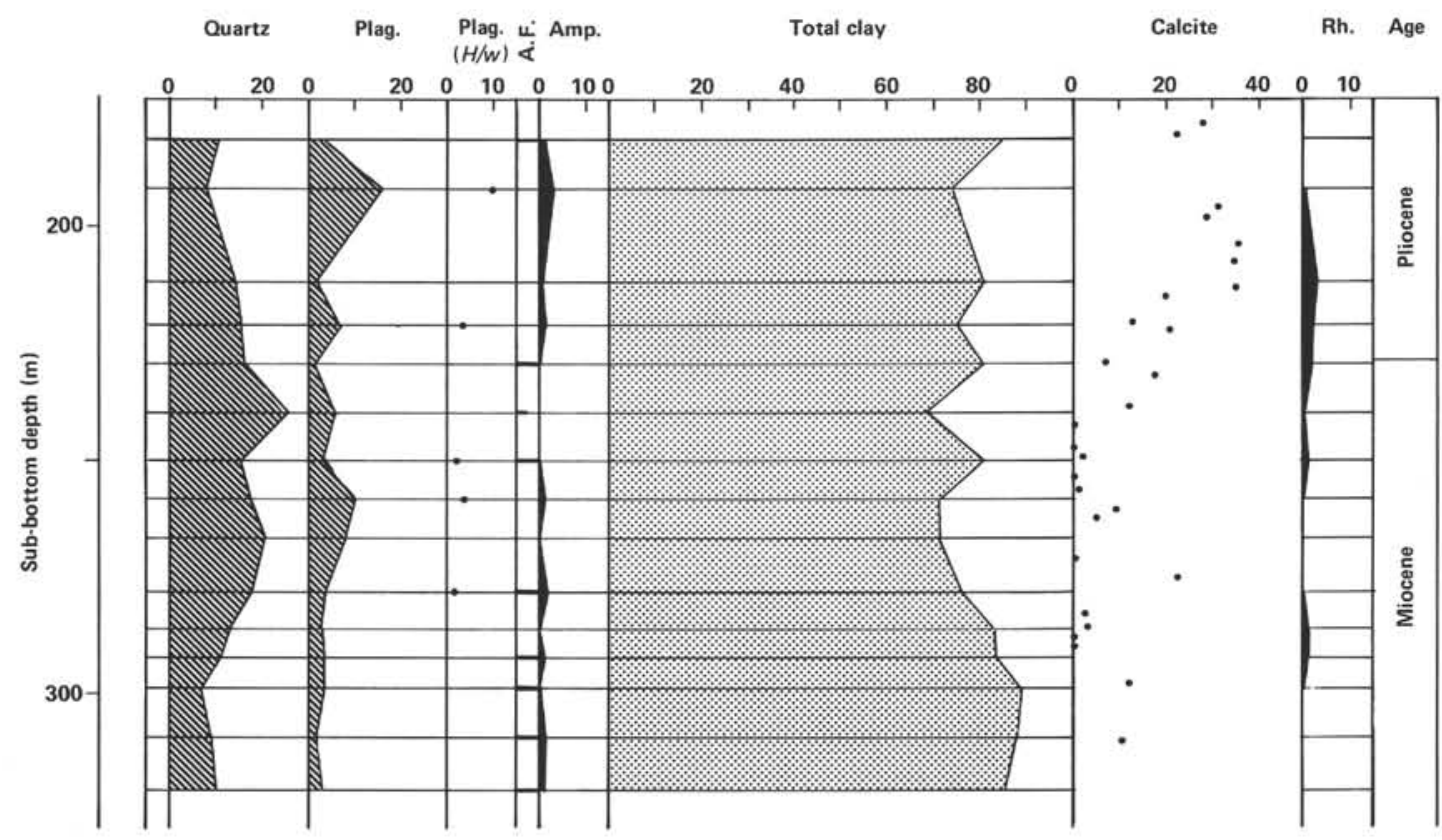

Figure 5. Site 542, mineralogy of bulk samples. (See Fig. 3 for explanation of symbols.)

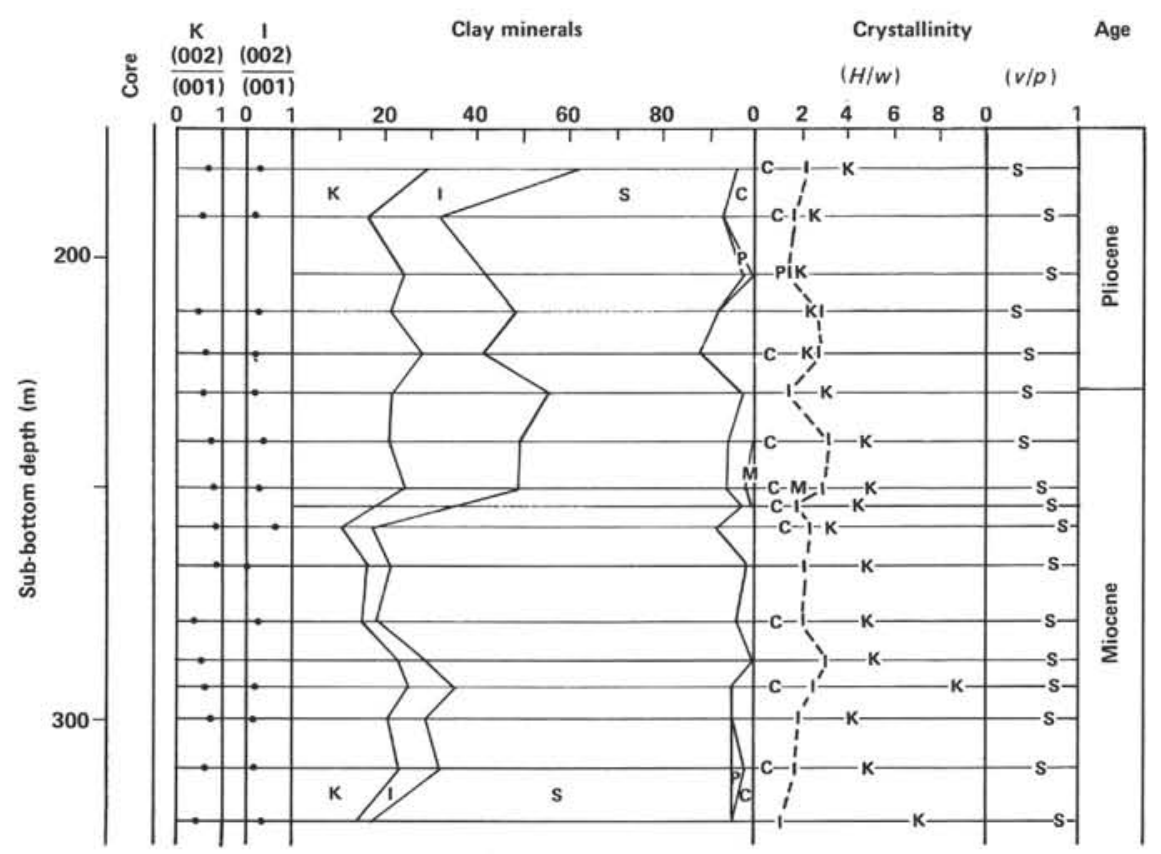

Figure 6. Site 542, mineralogy of $<2-\mu \mathrm{m}$ fraction samples. (For an explanation of the symbols see Fig. 4.)

1976): it is very commonly associated with opal-CT (Stonecipher, 1976; Fig. 6). With increasing time clinoptilolite may eventually be converted to K-feldspar, which does in fact appear at the very base of Hole 543A (Hay, 1966; Fig. 7). However, K-feldspar is also an authigenic replacement of calcic plagioclase phenocrysts in basalts immediately beneath these sediments (see Natland, this volume; and Bougault, et al., this volume) and therefore may reflect diagenetic conditions that also modified the sediments.

\section{Opal-CT}

Opal-CT is formed by crystallization of amorphous silica that is biogenic (or volcanic) in origin. On X-ray diffractograms it is characterized by a broad peak centered at $21.8^{\circ} 2 \theta$ (Fig. 2D), commonly with a small "shoulder" on the low $2 \theta$ side resulting from some tridymite stacking in the opal. X-ray amorphous opal-A can give a very broad hump in about the same position (Jones and Segnit, 1971), but this was not seen in the 


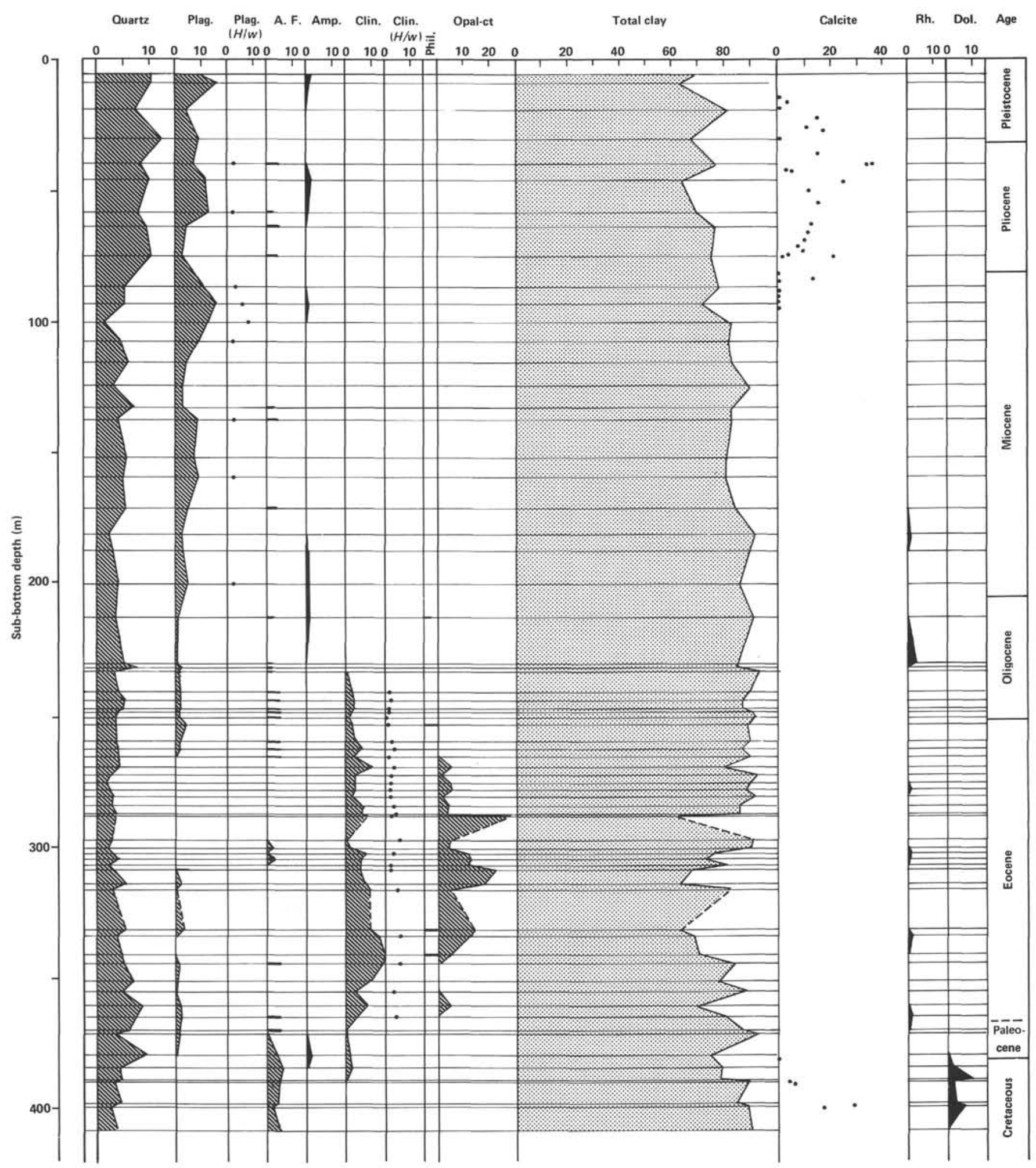

Figure 7. Site 543, mineralogy of bulk samples. (For an explanation of the symbols, see Fig. 3. Alkali feldspar was determined quantitatively below $380 \mathrm{~m}$ only; above $380 \mathrm{~m}$ only its presence was recorded.)

Leg 78A samples. Biogenic amorphous silica transforms in due course to opal-CT and then to quartz (e.g., Pisciotto 1981). The controls on the time taken for these diagenetic reactions include temperature, $\mathrm{pH}$, and the concentration of magnesium and hydroxyl ions (Kast- ner, Keene, and Gieskes 1977; Kastner and Gieskes, in press).

At Site 543 the occurrence of opal-CT is clearly related to that of radiolarians, in that the deepest level of both is at $330 \mathrm{~m}$. However, the stratigraphically higher 


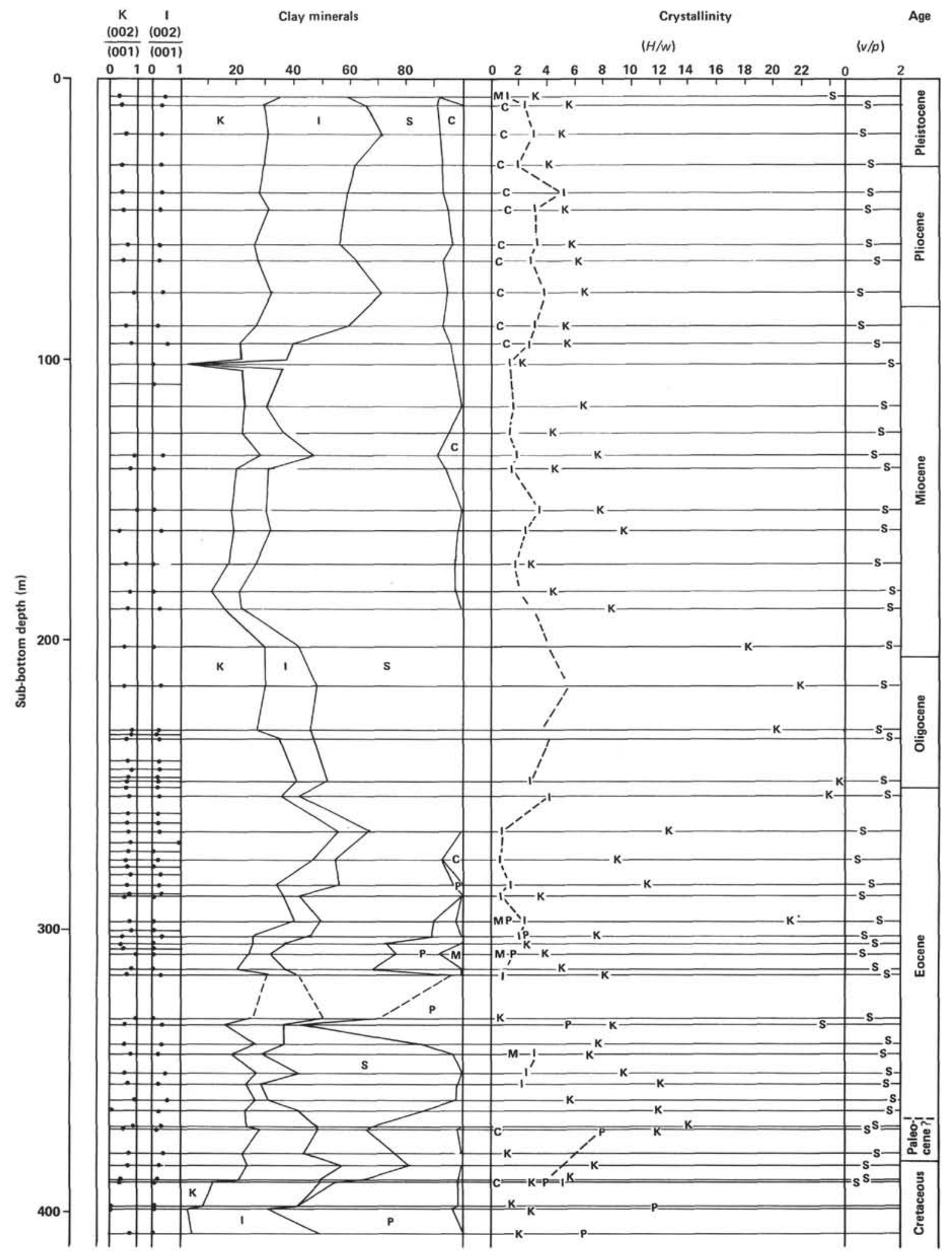

Figure 8. Site 543 , mineralogy of $<2-\mu \mathrm{m}$ fraction samples. (See Fig. 4 for an explanation of the symbols.)

levels of the radiolarian muds do not contain opal-CT: the transformation from opal-A is very slow at low temperatures and has apparently required the $40 \mathrm{Ma}$ since the late Eocene to take place. Scanning electron micrographs reveal opal-CT in the form of lepispheres grow- ing on radiolarian tests (Plate 1, Figs. 1 and 2). These radiolarians do not seem significantly dissolved, though many are broken (N. Renz, personal communication, 1982); they may be important as sites of nucleation as well as sources of silica. The silica in the lepispheres 


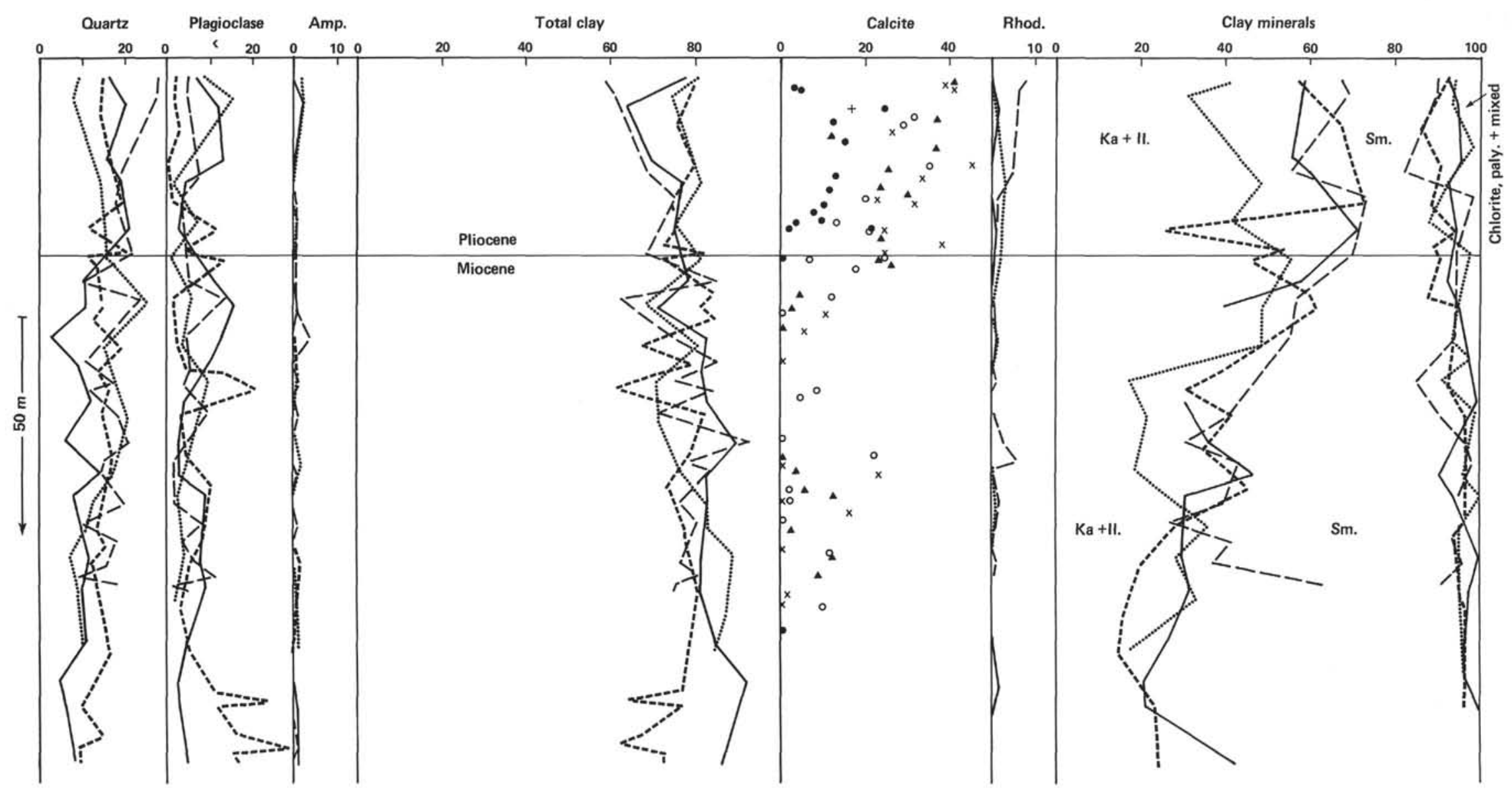

Figure 9. Comparison of mineralogy of Miocene sections cored at Sites 541, 542, and 543. (Plag. = plagioclase, Amp. = amphibole. In the clay minerals column only smectite is plotted separately. Kaolinite + illite are on the left, chlorite + palygorskite + mixed-layer clays are on the right.) 
may have been derived from very delicate parts of the skeletons such as spines or from less robust species of radiolarians that have now completely dissolved.

Opal-CT is most abundant where there are both radiolarians and palygorskite. Palygorskite is a magnesiumrich clay, and whiskers of it may have been additional sites of silica nucleation. Further transformation of opalCT to quartz is suggested by the increase in quartz content of the sediments below $330 \mathrm{~m}$. The presence of carbonate below $380 \mathrm{~m}$ has no particular effect on the proportions of silica minerals (contrast Lancelot, 1973).

\section{Dolomite}

Dolomite, conspicuous as scattered rhombs in smear slides as well as in diffractograms of the basal sediments, is euhedral and almost certainly authigenic (Plate 1, Fig. 3). Calcareous nannofossils in these lowest cores show strong dissolution (Bergen, this volume) and probably acted as a source of calcium. The extra magnesium source in this near-ridge setting could have been hydrothermal or a result of alteration of basalt.

\section{Authigenic Clays-Smectite and Palygorskite}

Whereas the poorly crystalline (low $v / p$ ) smectite in the near-surface sediments may be detrital, the abundant and well-crystallized smectite in the Miocene and older deposits is probably authigenic. It may have been derived both by neoformation of detrital smectite (Dunoyer de Segonzac, 1970) and by alteration of unstable volcanic debris.

Palygorskite was first reported from deep-sea sediments by Bonatti and Joensuu (1968) and has since been described from several Atlantic DSDP sites, for example, Site 12 (Peterson, Edgar et al., 1970), Site 119 (Laughton, Berggren, et al., 1972), Sites 135 to 140 , (von Rad and Rösch, 1972), Sites 327 and 328 (Barker, Dalziel, et al., 1977), Sites 332 to 335 (Aumento, Melson, et al., 1977), Site 398 (Sibuet, Ryan, et al., 1979), Sites 415 and 416 (Lancelot, Winterer, et al., 1980) and Sites 417 and 418 (Mann and Müller, 1980c). At Sites 398,415 , and 416 palygorskite was thought to be detrital but it has otherwise been considered authigenic. An unusual occurrence in a dredge haul quite near Site 543 was interpreted by Bowles et al. (1971) to have been precipitated directly from hydrothermal solution. Although palygorskite has not been synthesized and little is known of its genesis, it is believed to form during the alteration of volcanic glass, via a smectite intermediate:

$$
\begin{aligned}
\text { Smectite + silica }+(\mathrm{Ca}, \mathrm{Mg}) & \rightarrow \text { palygorskite } \\
& + \text { clinoptilolite (Bonatti } \\
& \text { and Joensuu, 1968) }
\end{aligned}
$$

Silica may be derived from (1) alteration of silicic ashes, (2) dissolution of biogenic opal, and (3) rivers draining regions of lateritic weathering (von Rad and Rösch, 1972).

Calcium and magnesium are derived from seawater: magnesium may also be hydrothermal, or derived from alteration of serpentine. The formation of palygorskite rather than sepiolite implies that $\left(\mathrm{Mg}^{++}\right)$and $\left(\mathrm{Al}^{+++}\right)$ must have been particularly high. An origin from degraded smectite is consistent with the observed antithetic relation in the abundance of the two minerals (Fig. 8). Scanning electron micrographs reveal the typical fibrous habit of palygorskite (Plate 1, Figs. 3 and 4).

\section{Evolution of the Sedimentary Sequence}

The sedimentary and diagenetic history of Site 543 as it passed from ridge crest through open ocean to a neararc setting is summarized in Fig. 10.

\section{Effect of Mineralogy on Physical Properties}

An interesting feature is apparent from a plot of physical properties against mineralogy for Site 543 (Fig. 11). The proportion of smectite has a strong effect on density, porosity, water content, and shear strength. This effect is not surprising, as smectite is an expandable mineral, able to take up a variable quantity of water between the layers: thus the smectite-rich muds are less dense, more porous, wetter, and weaker than the underlying radiolarian muds. None of the other minerals has a very marked effect on physical properties, except that quartz-rich sediments at the top of the section are relatively dense. It is curious that the opal-CT-rich muds have such similar physical properties to the lithologies above and below. Core recovery was rather patchy in the cristobalitic interval, which may be linked with the incipient formation of chert.

At Sites 541 and 542 the variation in amount of smectite is related to the amount and style of deformation noted in the cores (see Cowan et al., this volume). In particular, the smectite-rich intervals below $380 \mathrm{~m}$ at Site 541 and below $250 \mathrm{~m}$ at Site 542 correspond exactly to the zones of pervasively sheared, scaly clays. Similarly, brecciation in Tectonic Unit A of Site 541 corresponds to the increase in smectite below $230 \mathrm{~m}$. It is possible that very high pore fluid pressures in subduction zones may affect the amount of water within the crystal structure, and the presence of expandable clays may be crucial in the formation of décollement zones.

\section{CONCLUSIONS AND SPECULATIONS}

The sediments cored at Site 543 correspond quite well to the distribution patterns of deep North Atlantic sediments described by Thiede et al. (1981). The only differences are in the Neogene sediments, and these are readily explained by the increasing proximity of the depositional sites to volcanic arc sources at that time. Thiede et al. report a high proportion of terrigenous components from 70 to $50 \mathrm{Ma}$ and 30 to $10 \mathrm{Ma}$, high biogenic silica from 50 to $40 \mathrm{Ma}$, and moderately high calcite from $25 \mathrm{Ma}$ onwards, for basins of about $5.5 \mathrm{~km}$ depth. These variations are related to organic productivity, dissolution rates of calcite and opal (largely functions of temperature and dissolved oxygen), and sediment supply from the continents (a function of climate, tectonic processes, and sea level).

The décollement zone, which divides subducted from accreted sediment, is at the boundary between smectiterich and radiolarian-rich, smectite-poor muds. Thus in the Leg $78 \mathrm{~A}$ area the sediments mainly derived from the 


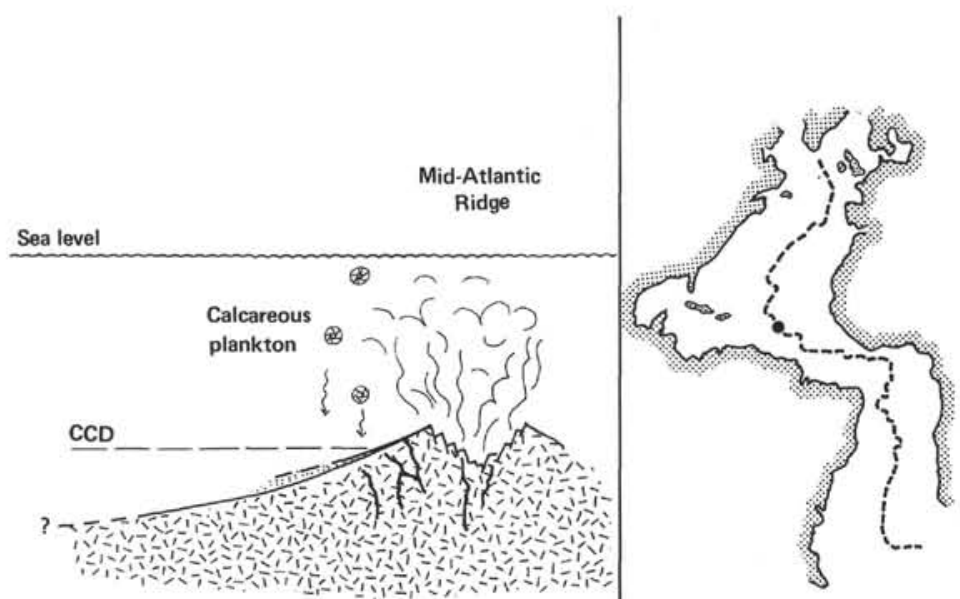

$75 \mathrm{Ma}$

No terrigenous input
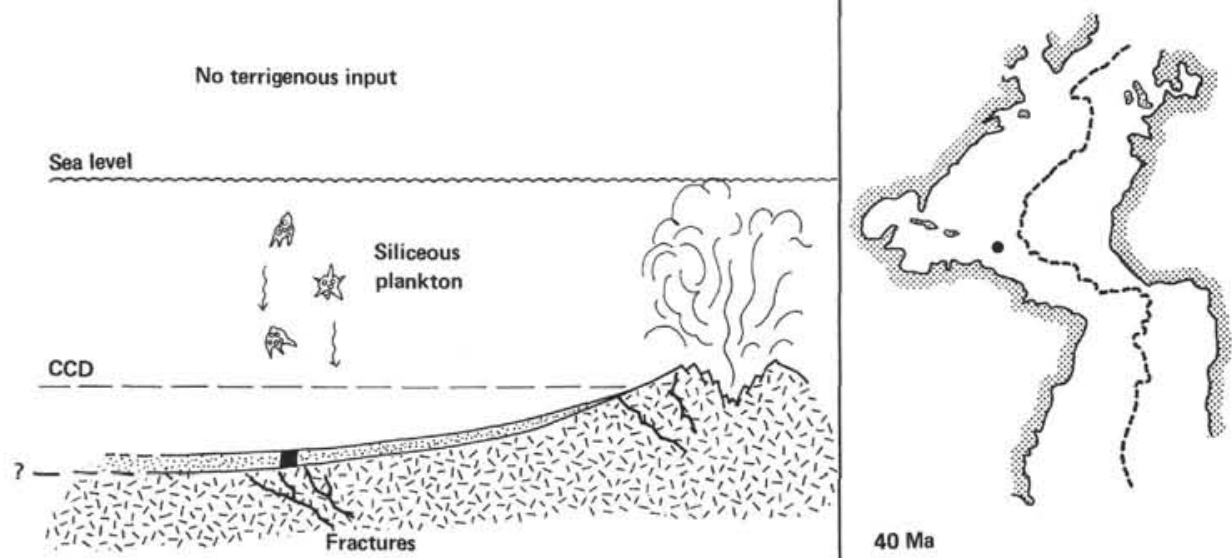

$40 \mathrm{Ma}$

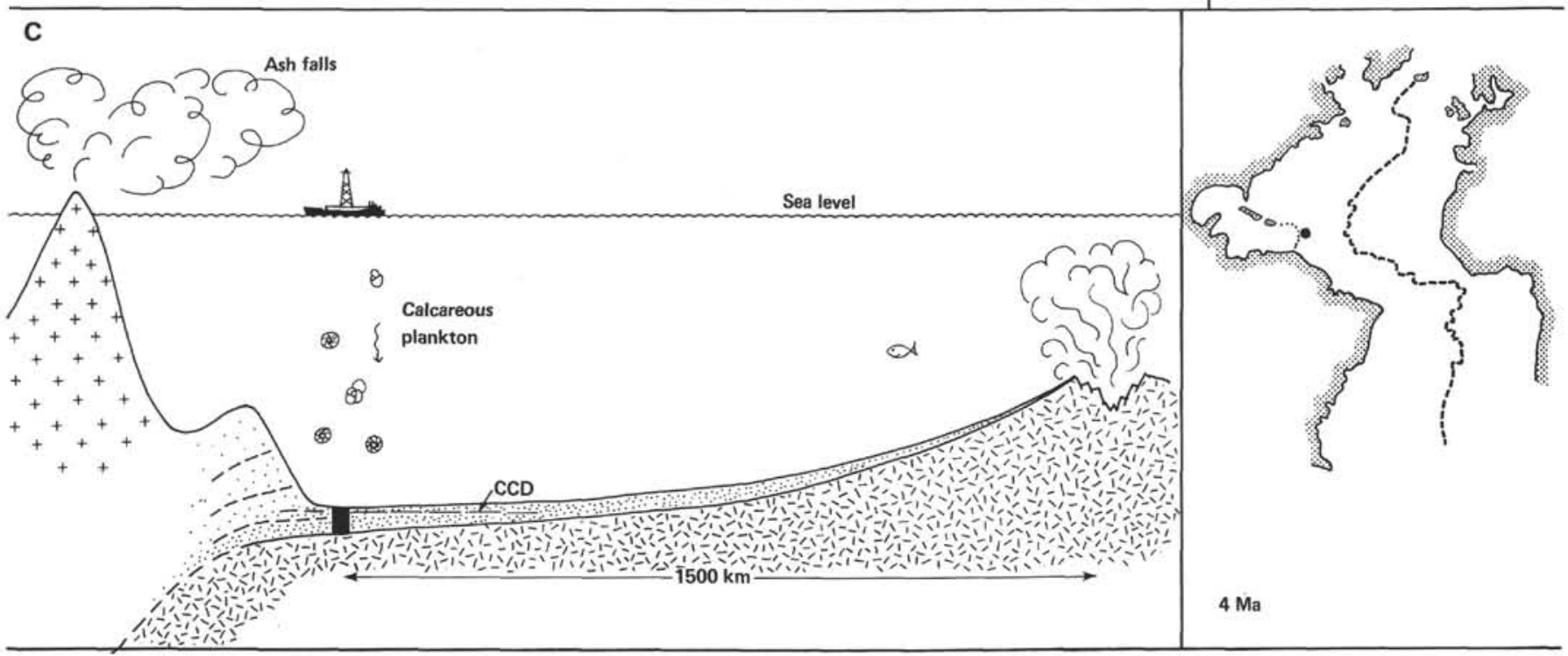

Figure 10. Evolution of Site 543. (Oceanic sediments shown in stipple, Site 543 in black. Vertical exaggeration $1000 \times$. Insets show Atlantic reconstructions [from Berger and Winterer, 1974] with the site marked. A. Campanian, 75 Ma. (Ridge-crest setting; iron and manganese-rich precipitates, hydrothermal activity, alteration of basalts.) B. Late Eocene, $40 \mathrm{Ma}$. (Open ocean [red clay facies] below CCD. Formation of zeolites. Deposition of opaline silica.) C. Pliocene, $4 \mathrm{Ma}$. (Glomar Challenger in her future position. Diagenesis of amorphous silica to opal-CT, zeolites to alkali feldspar. Neoformation of clays, alteration of volcanic glass.) 


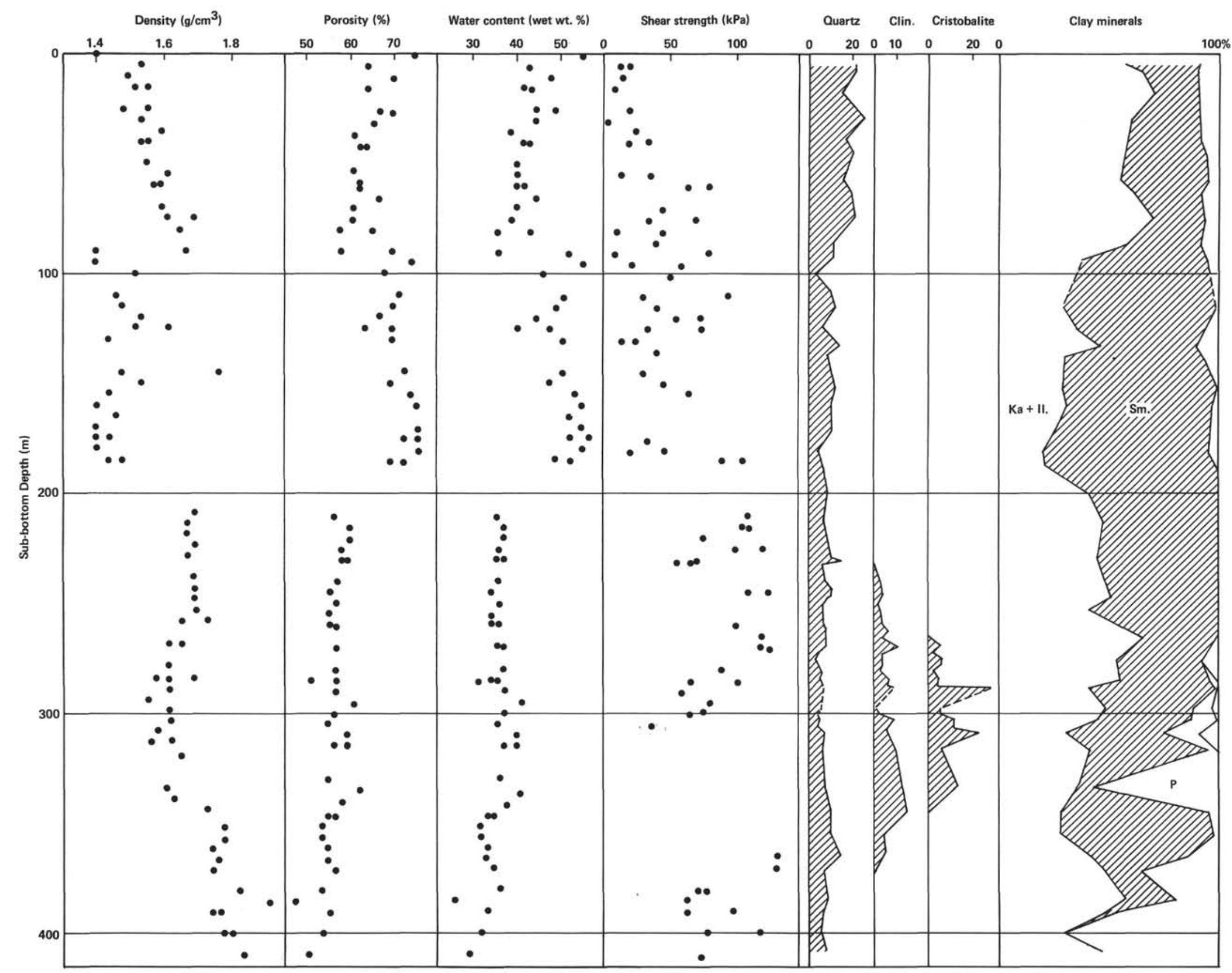

Figure 11. Physical properties and principal mineralogy of sediments from Site 543. (See text for discussion. Ka. $=$ kaolinite, Sm. $=$ smectite, Il. $=$ illite, and $\mathrm{P}=$ palygorskite. 
arc, that is, the Neogene ashy muds, are not recycled in the subduction zone. It is the muds, derived from openocean, continent, or ocean-crust sources, that go down, perhaps to be partially melted and contribute to arc magmas. In general, if the composition of arc magmas is to some extent related to that of subducting oceanic sediment, it might be possible to link temporal variations in arc chemistry to changing hydrographic-tectonic conditions in the ocean.

\section{ACKNOWLEDGMENTS}

I am grateful to The Natural Environment Research Council (NERC) for financial support and to Professor J. Tarney for laboratory facilities during the shore-based study. I thank Rob Wilson and George McTurk for technical assistance and my colleagues at Leicester for useful discussion. The manuscript was improved by comments from Drs. H. G. Reading, J. D. Hudson, J. R. Hein, and J. Schoonmaker. Dr. M. Kastner advised on silica diagenesis. Janet Wood typed the final manuscript.

\section{REFERENCES}

Aumento, F., Melson, W. G., et al., 1977. Init. Repts. DSDP, 37: Washington (U.S. Govt. Printing Office).

Barker, P., Dalziel, I. W. D., et al., 1977. Init. Repts. DSDP, 36: Washington (U.S. Govt. Printing Office).

Berger, W. H., and Winterer, E. L., 1974. Plate stratigraphy and the fluctuating carbonate line. In Hsü, K. J., and Jenkyns, H. C. (Eds.), Pelagic Sediments on Land and Under the Sea: London (Blackwell Sci. Publ. Int. Assoc. Sediment.), 1:11-48.

Biscaye, P. E., 1965. Mineralogy and sedimentation of recent deep-sea clay in the Atlantic Ocean and adjacent seas and oceans. Geol. Soc. Am. Bull., 76:803-832.

Bonatti, E., and Joensuu, O., 1968. Palygorskite from Atlantic deepsea sediments. Am. Mineral., 53:975-983.

Bowles, F. A., Angino, E. A., Hosterman, J. W., and Galle, O.K. 1971. Precipitation of deep-sea palygorskite and sepiolite. Earth Planet. Sci. Lett., 11:324-332.

Brindley, G. W., and Brown, G. (Eds.), 1980. Crystal structures of clay minerals and their X-ray identification. Min. Soc. London Monogr. 5.

Calvert, S. E., 1974. Deposition and diagenesis of silica in marine sediments. In Hsü, K. J., and Jenkyns, H. C. (Eds.), Pelagic Sediments on Land and Under the Sea: London (Blackwell Sci. Publ. Int. Assoc. Sediment.), 1:273-279.

Dunoyer de Segonzac, G., 1970. The transformation of clay minerals during diagenesis and low-grade metamorphism - a review. Sedimentology, 17:281-340.

Gibson, T. G., and Towe, K. M., 1971. Eocene volcanism and the origin of Horizon A. Science, 172:152-154. 1975. Origin of Horizon A: Clarification of viewpoint. Science, 188:1221.

Hathaway, J. C., and Sachs, P. L., 1965. Sepiolite and clinoptilolite from the Mid-Atlantic Ridge. Am. Mineral., 50:852-867.

Hay, R. L., 1966. Zeolites and zeolitic reactions in sedimentary rocks. Geol. Soc. Am. Spec. Pap. 85.

Jones, J. B., and Segnit, E. R., 1971. The nature of opal. I. Nomenclature and constituent phases. J. Geol. Soc. Aust., 18:57-68.
Kastner, M., and Gieskes, J. M., in press. Opal-A to opal-CT transformation: a kinetic study. In Ijima, F., and Siever, R. (Eds.), Developments in Sedimentology: New York (Elsevier).

Kastner, M., Keene, J. B., and Gieskes, J. M., 1977. Diagenesis of siliceous oozes-1. Chemical controls on the rate of opal-A to opalCT transformation-an experimental study. Geochim. Cosmochim. Acta, 41:1041-1059.

Lancelot, Y., 1973. Chert and silica diagenesis in sediments from the Central Pacific. In Winterer, E. L., Ewing, J. I., et al., Init. Repts. DSDP, 17: Washington (U.S. Govt. Printing Office), 377-405.

Lancelot, Y., Winterer, E. L., et al., 1980. Init. Repts. DSDP, 50: Washington (U.S. Govt. Printing Office).

Laughton, A. S., Berggren, W. A., et al., 1972. Init. Repts. DSDP, 12: Washington (U.S. Govt. Printing Office).

Lonsdale, P. F., 1975. Sedimentation and tectonic modification of the Samoan archipelagic apron. Am. Assoc. Petrol. Geol. Bull., 59: 780-798.

Mann, V., and Müller, G., 1980a. Composition of sediments of the Japan Trench transect, Legs 56 and 57, DSDP. In Scientific Party, Init. Repts. DSDP, 56, 57, Pt. 2: Washington (U.S. Govt. Printing Office).

1980b. Mineralogy of the sedimentary sections encountered on Leg 55 (Sites 430 through 433) based on X-ray diffraction. In Jackson, E. D., Koisumi, I., et al., Init. Repts. DSDP, 55: Washington (U.S. Govt. Printing Office), 857-859.

1980c. X-ray mineralogy of DSDP Legs 51 through 53, western North Atlantic. In Donnelly, T., Francheteau, J., Bryan, W., Robinson, P., Flower, M., Salisbury, M., et al., Init. Repts. $D S D P, 51,52,53$, Pt. 2: Washington (U.S. Govt. Printing Office), 721-730.

Pisciotto, K. A., 1981. Diagenetic trends in the siliceous facies of the Monterey Shale in the Santa Maria region, California. Sedimentology, 28:547-571.

von Rad, U., and Rösch, H., 1972. Mineralogy and origin of clay minerals, silica and authigenic silicates in Leg 14 sediments. In Hayes, D. E., Pimm, A. C., et al., Init. Repts. DSDP, 14: Washington (U..S Govt. Printing Office), 727-751.

Rex, R. W., and Murray, B., 1970. X-ray mineralogy studies, Leg 4; and Appendix III. In Bader, R. G., Gerard, R. D., et al., Init. Repts. DSDP, 4: Washington (U.S. Govt. Printing Office), 325-326 and 748-753.

Sibuet, J.-C., Ryan, W. B. F., et al., 1979. Init. Repts. DSDP, 47, Pt. 2: Washington (U.S. Govt. Printing Office).

Stonecipher, S. A., 1976. Origin, distribution and diagenesis of phillipsite and clinoptilolite in deep-sea sediments. Chem. Geol., 17: 307-318.

Thiede, J., Strand, J. E., and Agdestein, T., 1981. The distribution of major pelagic sediment components in the Mesozoic and Cenozoic North Atlantic Ocean. In Warme, J. E., Douglas, R. G., and Winterer, E. L., (Eds.), The Deep Sea Drilling Project: A Decade of Progress: Soc. Econ. Paleontol. Mineral. Spec. Publ., 32:67-90.

van Zinderen Bakker, E. M., 1978. Late Mesozoic and Tertiary paleoenvironments of the Sahara region. In van Zinderen Bakker, E. M. (Ed.), Antarctic Glacial History and World Paleoenvironments: Rotterdam (A. A. Balkema), pp. 129-135.

Date of Initial Receipt: May 27, 1982

Date of Acceptance: March 3, 1983 


\section{J. PUDSEY}
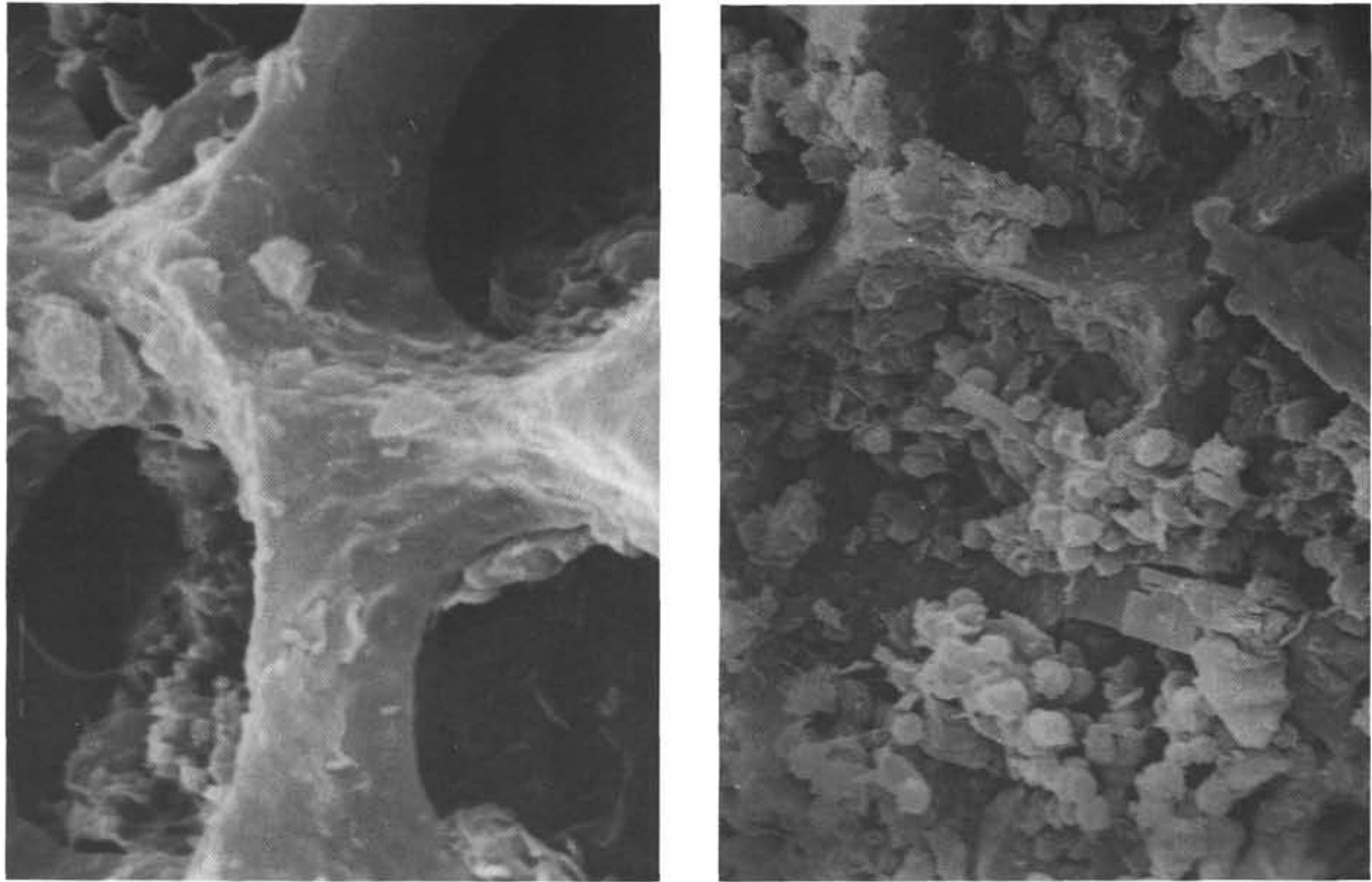

2
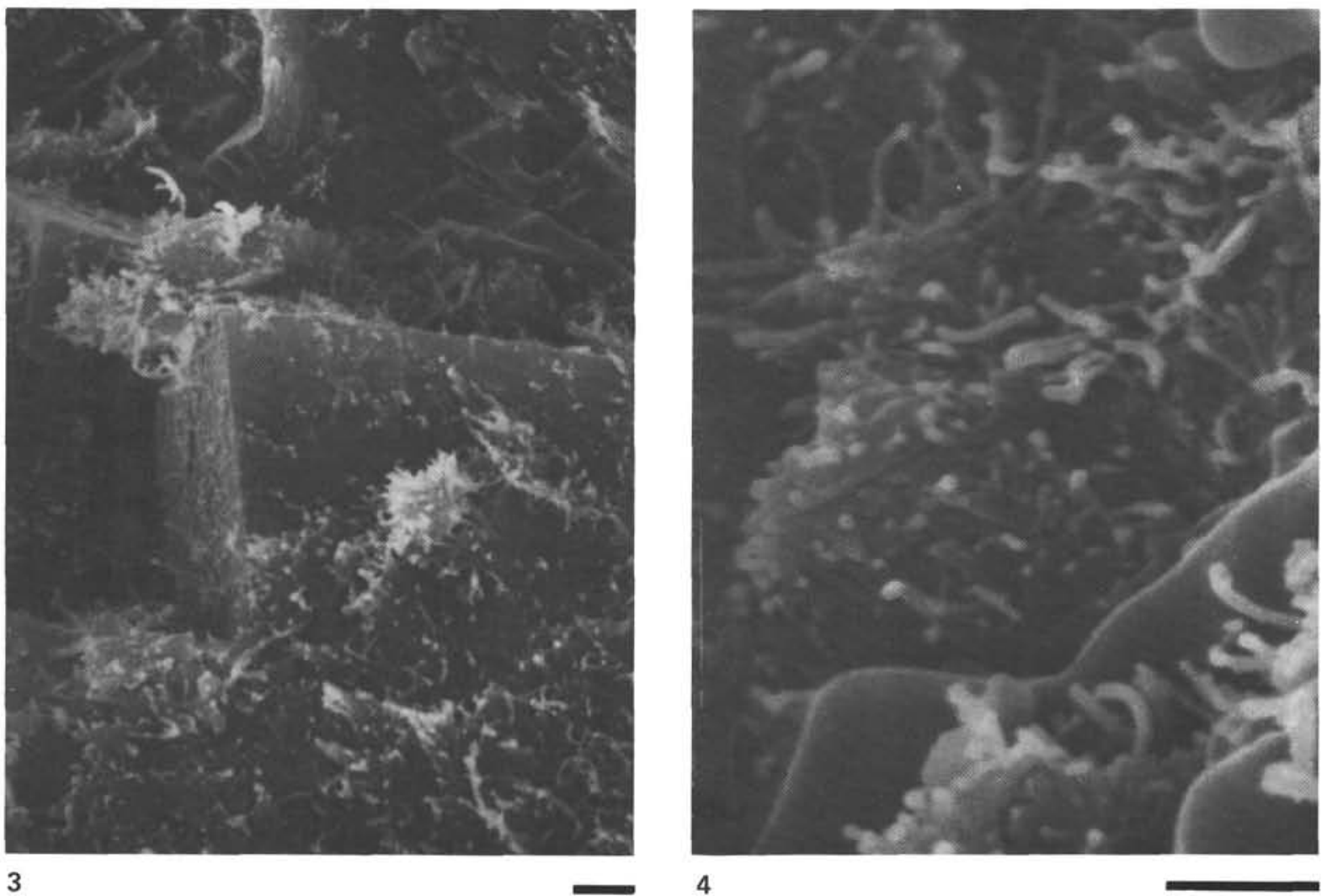

Plate 1. Scanning electron micrographs showing authigenic minerals. 1. Part of a radiolarian test from the Miocene. (Diagenesis has not significantly affected this sample. Sample 541-50,CC. Scale bar is $1 \mu \mathrm{m}$.) 2. Part of a radiolarian test from the upper Eocene. (Extensive silica precipitation in the form of lepispheres of opal-CT. Compare the corroded radiolarian with that shown in Fig. 1. Sample 543-31-2, $8 \mathrm{~cm}$. Scale bar is $5 \mu \mathrm{m}$.) 3. Authigenic dolomite (rhombs) and palygorskite (fibers) from the Cretaceous. (Sample 543A-9-1, $38 \mathrm{~cm}$. Scale bar is $1 \mu \mathrm{m}$.) 4. Authigenic palygorskite fibers from the Cretaceous. (Curved object is part of a calcareous microfossil on which some of the fibers have nucleated. Sample $543 \mathrm{~A}-9-1,38 \mathrm{~cm}$. Scale bar is $1 \mu \mathrm{m}$.) 